\title{
Generalizations of the Sampling Theorem: Seven Decades After Nyquist
}

\author{
P. P. Vaidyanathan, Fellow, IEEE
}

\begin{abstract}
The sampling theorem is one of the most basic and fascinating topics in engineering sciences. The most well- known form is Shannon's uniform-sampling theorem for bandlimited signals. Extensions of this to bandpass signals and multiband signals, and to nonuniform sampling are also well-known. The connection between such extensions and the theory of filter banks in DSP has been well established. This paper presents some of the less known aspects of sampling, with special emphasis on non bandlimited signals, pointwise stability of reconstruction, and reconstruction from nonuniform samples. Applications in multiresolution computation and in digital spline interpolation are also reviewed.
\end{abstract}

Index Terms-Bandlimited signals, FIR reconstruction, multiresolution, nonuniform sampling, sampling theorems.

\section{INTRODUCTION}

$\mathbf{T}$ HE sampling theorem is one of the most basic and fascinating topics in engineering sciences. The most wellknown form is the uniform sampling theorem for bandlimited signals, due to Nyquist and Shannon [9], [13]. This has also been attributed to Whittaker and Cauchy (see [6]). It is the fundamental tool that allows the processing of real signals using digital signal processors (DSP). Extensions of this to bandpass signals and multiband signals, and to nonuniform sampling are also well-known [6], [22]. The connection between such extensions and the theory of filter banks in DSP has also been well established (e.g., see Chap. 10 in [23]). Further novel extensions can be found in [5]. This paper is a review of some of the less known aspects of sampling, with special emphasis on non bandlimited signals, stability of reconstruction, and reconstruction from nonuniform samples. [24]-[27].

\section{A. Outline}

In Section II, we consider sampling theorems for non bandlimited signals. These are often referred to as multiresolution (MR) or wavelet sampling theorems. An application in digital interpolation is reviewed in Section III, and demonstrated with $B$-spline interpolation of images. Stability of the reconstruction process is defined in Section IV and addressed in considerable detail there. We explain the importance of pointwise stability as opposed to stability in terms of energy. Such pointwise stability is

\footnotetext{
Manuscript received April 16, 2000; revised March 23, 2001. This work was supported in part by the National Science Foundation under grant MIP-0703755, in part by the Office of Naval Research under grant N00014-99-1-1002, and in part by Microsoft Research, Redmond, WA. This paper has been presented in parts at conferences This paper was recommended by Associate Editor M. O. Ahmad.

The author is with the Department of Electrical Engineering, California Institute of Technology, Pasadena, CA 91125 USA (e-mail: ppvnath@sys.caltech.edu).

Publisher Item Identifier S 1057-7122(01)07720-0.
}

then established for the cases of bandlimited as well as nonbandlimited sampling in Sections IV-B and C. The connection between this kind of stability and functional subspaces is reviewed in Section IV-D. The role of nonuniform sampling in MR theory is explained in Section V. We show in particular that the MR coefficients can often be calculated using FIR filtering operations without the use of oversampling (which is often resorted to). Sampling theorems for non bandlimited discrete time signals are discussed in Section VI. These results are based on well established concepts in multirate digital signal processing. Results on FIR reconstructibility in this context are established in Sections VI-B-D. Certain basic properties satisfied by bandlimited signals are summarized in the Appendices for convenience.

\section{B. Notations}

Unless mentioned otherwise, all notations are as in [23]. The term $\sigma$-BL refers to signals that are bandlimited to $|\omega|<\sigma$ (i.e., Fourier transform is zero outside). We use the notations $[x(n)]_{\downarrow M}$ and $[X(z)]_{\downarrow M}$ to denote the decimated version $x(M n)$ and its $z$-transform. The expanded version

$$
\begin{cases}x\left(\frac{n}{M}\right), & n=\text { mul of } M \\ 0, & \text { otherwise }\end{cases}
$$

is similary denoted by $[x(n)]_{\uparrow M}$, and its $z$-transform $X\left(z^{M}\right)$ denoted by $[X(z)]_{\uparrow M}$. In situations where the $z$-transform does not exist in the conventional sense (e.g., ideal filters), the notation $z$ stands for $e^{j \omega}$ so that $H(z)$ is the frequency response $H\left(e^{j \omega}\right)$. The type 1 and type 2 polyphase representations of $H(z)$ with respect to an integer $M$ are given by [23]

$$
H(z)= \begin{cases}\sum_{k=0}^{M-1} z^{-k} E_{k}\left(z^{M}\right) & \text { (type } 1 \text { polyphase) } \\ \sum_{k=0}^{M-1} z^{k} R_{k}\left(z^{M}\right) & \text { (type } 2 \text { polyphase) }\end{cases}
$$

The type used is usually clear from the context and is therefore often not mentioned.

BIBO stability stands for bounded input bounded output stability [10]. A sequence $h(n)$ is an $\ell_{1}$ sequence if $\sum_{n}|h(n)|<$ $\infty$ and it is an $\ell_{2}$ sequence if $\sum_{n}|h(n)|^{2}<\infty$. A linear time invariant filter is BIBO stable if and only if the impulse response $h(n)$ is in $\ell_{1}$. For continuous time signals similar definitions ( $L_{1}$ and $L_{2}$ ) apply with sums replaced by integrals. Note that $\ell_{1} \subset \ell_{2}$, but we do not have $L_{1} \subset L_{2}$.

\section{NONBANDLIMITED SigNALS}

If we have the apriori information that a signal is bandlimited to a known region, we can recover it from appropriately-spaced samples by filtering. If a signal is not bandlimited, can we still recover it from samples? The answer depends on what other 


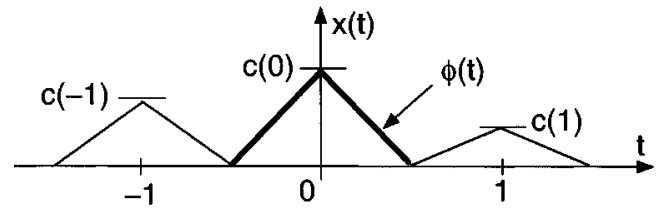

Fig. 1. A nonbandlimited signal.

apriori information we have. For example, suppose we have the knowledge that $x(t)$ has the form

$$
x(t)=\sum_{k=-\infty}^{\infty} c(k) \phi(t-k) \quad \text { (signal model) }
$$

where $\phi(t)$ is a known function. Denoting the continuous time Fourier transforms (FT) of $x(t)$ and $\phi(t)$ as $X(j \omega)$ and $\Phi(j \omega)$, and the discrete time FT of $c(n)$ as $C\left(e^{j \omega}\right)$, we equivalently have $X(j \omega)=C\left(e^{j \omega}\right) \phi(j \omega)$. As a first example, assume that $\phi(t)$ is a time-limited signal as demonstrated in Fig. 1. In this example, the samples of $x(t)$ at integer points are $x(n)=c(n)$. So we can trivially reconstruct $x(t)$ from its samples using the formula

$$
x(t)=\sum_{k=-\infty}^{\infty} x(k) s(t-k)
$$

where $s(t)=\phi(t)$. More generally, this holds if $\phi(t)$ has the zero-crossing property $\phi(n)=\delta(n)$, i.e.,

$$
\phi(0)=1, \quad \phi(n)=0 \text { for other integers } n \text {. }
$$

Thus reconstruction from samples has been possible inspite of aliasing due to nonbandlimitedness.

A function $\phi(t)$ satisfying the zero-crossing property (3) is also referred to as a Nyquist(1) function in the literature. The argument "(1)" signifies that the zero crossings are separated by one unit of time. A special case is the example where $\phi(t)$ is the sine funtion

$$
\phi(t)=\frac{\sin \pi t}{\pi t}
$$

Since this is $\pi$-BL, the sum (1) is also $\pi$-BL. The sine function is Nyquist(1), so the reconstruction formula (2) holds. This corresponds to the familiar Shannon sampling and reconstruction.

If a function can be represented as in (1) where $\phi(t)$ is not Nyquist (Fig. 2), can we still reconstruct $x(t)$ from samples $x(n)$ ? The answer is yes for a large class of $\phi(t)$ as we now show. ${ }^{1}$ From (1) we see that the samples of $x(t)$ are given by

$$
x_{d}(n) \triangleq x(n)=\sum_{k=-\infty}^{\infty} c(k) \phi(n-k)
$$

which is nothing but a discrete-time convolution equation. Denoting the discrete time Fourier transforms of the sequences $x(n), c(n)$, and $\phi(n)$ by $X_{d}\left(e^{j \omega}\right), C\left(e^{j \omega}\right)$ and $\Phi_{d}\left(e^{j \omega}\right)$ we get

$$
X_{d}\left(e^{j \omega}\right)=C\left(e^{j \omega}\right) \Phi_{d}\left(e^{j \omega}\right)
$$

${ }^{1}$ Notice that the sample spacing is $T=1$, and that the sampling phase is such that $t=0$ is included. For a different sampling phase, the reconstruction conditions and equations have to be worked out again.
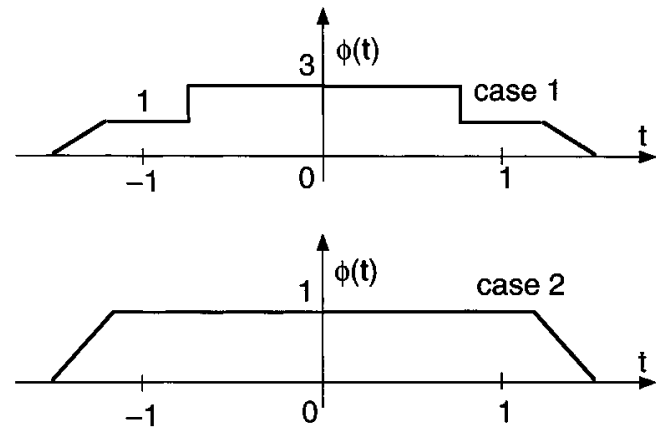

Fig. 2. Two non Nyquist choices of $\phi(t)$.

If $\Phi_{d}\left(e^{j \omega}\right) \neq 0$ for all $\omega$ we can write $C\left(e^{j \omega}\right)=X_{d}\left(e^{j \omega}\right) /$ $\Phi_{d}\left(e^{j \omega}\right)$. That is, we can identify $c(n)$ from $x(n)$ using

$$
c(n)=\sum_{k} x(k) \gamma(n-k)
$$

where $\gamma(n)$ is the of convolutional inverse of $\phi(n)$ i.e., its Fourier transform $\Gamma\left(e^{j \omega}\right)=1 / \Phi_{d}\left(e^{j \omega}\right)$. Recovery of $x(t)$ for all $t$ can then be done using (1). In summary, we have recovered $x(t)$ from the samples $x(n)$.

Return now to the examples in Fig. 2. We see that for the first function the nonzero samples of $\phi(n)$ are $\{1,3,1\}$ whereas for the second function these are $\{1,1,1\}$. In the first case $\left|\Phi_{d}\left(e^{j \omega}\right)\right|=3+2 \cos \omega>0$ whereas in the second case, $\left|\Phi_{d}\left(e^{j \omega}\right)\right|=|1+2 \cos \omega|=0$ at $\omega=2 \pi / 3$. So $x(t)$ is reconstrucible from $x(n)$ for the first case, but not the second. We conclude this section with a few remarks.

1) Discrete time model. The preceding discussion also shows this: given a discrete time signal $x_{d}(n)$ and an arbitrary function $\phi(t)$ we can almost always assume that $x_{d}(n)$ can be written in the form (5) for appropriate choice of $c(k)$, the only theoretical condition being that $\Phi_{d}\left(e^{j \omega}\right) \neq$ 0 for all $\omega$. In particular, we can regard $x_{d}(n)$ as samples of a continuous time signal $x(t)$ of the form $x(t)=$ $\sum_{k=-\infty}^{\infty} c(k) \phi(t-k)$.

2) Lack of shift invariance. For fixed $\phi(t)$, let $V_{0}$ denote the space of all signals which can be represented as in (1) for appropriate finite-energy $c(k)$. When $\phi(t)$ is the sine function we know that any shifted version of $x(t)$ (e.g., $x(t-0.1)$ ) also belongs to the space $V_{0}$ because time-shift does not affect bandlimitedness. For arbitrary $\phi(t)$ however, even though reconstruction from samples is often possible, the shifted versions of $x(t)$ do not in general belong to the same space $V_{0}$. This is readily verified with examples. For example shift $\phi(t)$ in Fig. 2 to obtain $\phi(t-0.1)$. This result cannot be expressed as a linear combination of the integer shifts $\{\phi(t-n)\}$.

3) Undersampling a wideband signal. As a special case of the signal model, suppose $\phi(t)$ is $2 \pi$-BL, that is, bandlimited to $-2 \pi<\omega<2 \pi$. Then $x(t)$ is also $2 \pi$-BL and the Shannonsampling rate would be $4 \pi$, implying the sample spacing $T=1 / 2$. Assuming for sake of argument that $\Phi(j \omega)$ is real and positive in $-2 \pi<\omega<2 \pi$, we see that $\Phi_{d}\left(e^{j \omega}\right)$ (aliased version of $\Phi(j \omega)$ ) is nonzero for 


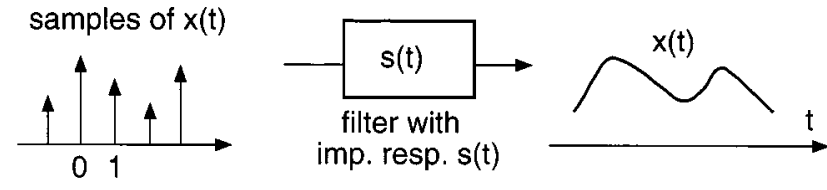

Fig. 3. Reconstruction of $x(t)$ from samples.

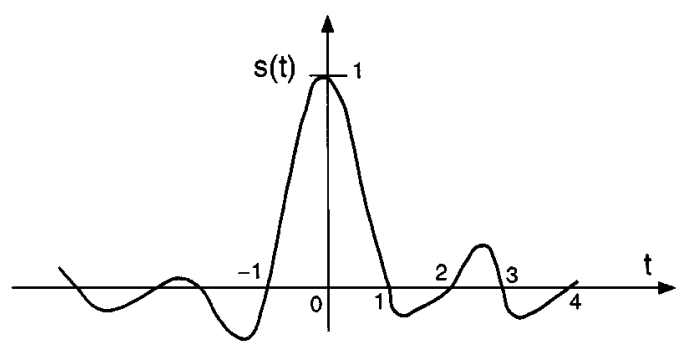

Fig. 4. Nyquist(1) or zero-crossing property of the reconstruction filter $s(t)$.

all $\omega$, and the reconstruction (7) is valid. Thus the model (1) allows a smaller sampling rate, that is, wider sample spacing of $T=1$, even though the Shannonrate spacing would be $T=1 / 2$.

4) Reconstruction filter. Substituting for $c(k)$ from (7) into (1) and simplifying we get

$$
x(t)=\sum_{i=-\infty}^{\infty} x(i) s(t-i)
$$

where $s(t)=\sum_{m=-\infty}^{\infty} \gamma(m) \phi(t-m)$ is the reconstruction filter. Thus, we simply pass the samples $x(n)$ through the continuous-time filter with impulse respose $s(t)$ as in standard D/A conversion. See Fig. 3.

From the preceding definition of the reconstruction filter $s(t)$ it follows that at the integer points $s(n)=\sum_{m=-\infty}^{\infty} \gamma(m) \phi(n-m)$. That is, $s(n)$ is obtained by passing $\phi(n)$ through its convolutional inverse $\gamma(n)$. Evidently therefore, $s(n)=\delta(n)$. That is, the continuous filter $s(t)$ has the Nyquist(1) property similar to the sine function $\sin \pi t / \pi t$ (Fig. 4).

5) MR spaces. Sampling theorems for signals of the type (1) are often known as wavelet or MR sampling theorems. Readers familiar with MR theory [8] will realize, however, that additional restrictions on $\phi(t)$ are required to generate a MR. Functions of the form $x(t)=\sum_{k} c(k) \phi(t-k)$ belong to the space $V_{0}$ in a MR decomposition. Roughly speaking, $V_{0}$ is like a subspace of lowpass signals with a certain degree of smoothness. The MR framework also defines finer subspaces $V_{k}$ (with higher bandwidth) and bandpass spaces $W_{k}$. If $\phi(t)$ were the sine function $\sin \pi t / \pi t$ the MR decomposition would be as in Fig. 5. In practice, $\phi(t)$ is not ideally bandlimited. So none of the subspaces represents ideal bandpass signals, even though they admit reconstruction from samples. These sampling theorems can also be extended to other subspaces like $W_{k}$ and arbitrary direct sums of such spaces [4].

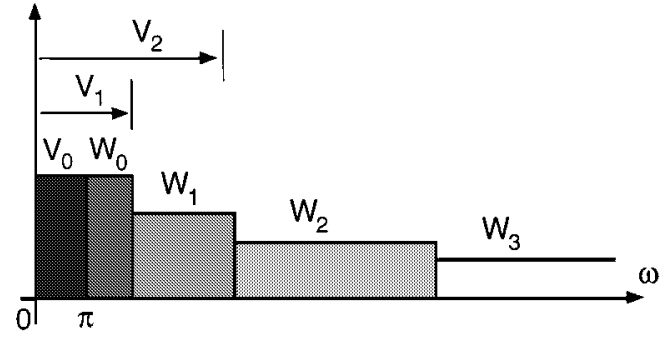

Fig. 5. MR decomposition based on ideal filters.

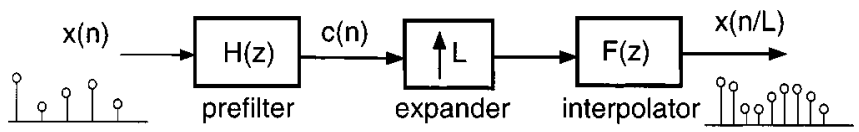

Fig. 6. Interpolation of a signal $x(n)$ with digital filters. The signal is assumed to have a continuous time model $x(t)=\sum_{k} c(k) \phi(t-k)$.

\section{APPLICATION IN INTERPOLATION}

As explained in Section II, a discrete time signal $x_{d}(n)$ can be viewed as a sampled version of $x(t)=\sum_{k} c(k) \phi(t-k)$. While true for almost any $\phi(t)$, this viewpoint is especially useful for certain choices of $\phi(t)$. For example, if $\phi(t)$ has smoothness properties such as a certain degree of differentiability everywhere, then we can use this to generate an interpolated version of $x(n)$. A $256 \times 256$ image can be displayed as a $512 \times 512$ image in this way (interpolation by two). Smoothness of $\phi(t)$ usually ensures that the interpolated result is visually pleasing (see example below). To see how the model can be used for interpolation notice that the samples of $x(t)$ at a finer spacing $1 / L$ are given by

$$
x\left(\frac{n}{L}\right)=\sum_{k=-\infty}^{\infty} c(k) \phi\left(\frac{n-k L}{L}\right)=\sum_{k=-\infty}^{\infty} c(k) f(n-k L)
$$

where $f(i)=\phi(i / L)$. In summary, we can reconstruct the finer samples $x(n / L)$ from $x(n)$ as shown in Fig. 6. First pass $x(n)$ through the digital prefilter $H\left(e^{j \omega}\right)=1 / \Phi_{d}\left(e^{j \omega}\right)$. This gives $c(n)$. Then, use the $L$-fold upsampler or expander [20] indicated as $\uparrow L$, followed by the interpolation filter $F(z)$, which is obtained by sampling $\phi(t)$ using the finer spacing $1 / L$. We see that the interpolation from $x(n)$ to $x(n / L)$ can be done entirely digitially. The function $\phi(t)$ is often chosen as a spline function. The use of cubic spline is especially common in image processing because its degree of differentiablity is two, which provides sufficient smoothness for the human visual system.

\section{A. Interpolation With Splines}

A spline $s(t)$ is a piecewise polynomial, with the pieces glued together at places $t_{k}$ called knots such that the function is differentiable a specified number of times even at the knots. The most commonly used splines have knots at integers, that is $t_{k}=k$, where $-\infty \leq k \leq \infty$.

Definition 1. Splines: An Nth-order spline with knots at integers (just " $N$ th-order spline" for the rest of the paper) is a function $s(t)$ such that

1) $s(t)$ is a polynomial of degree $N$ or less between integers, (i.e., in $k<t<k+1$ for all integer $k$ ). 
(a)

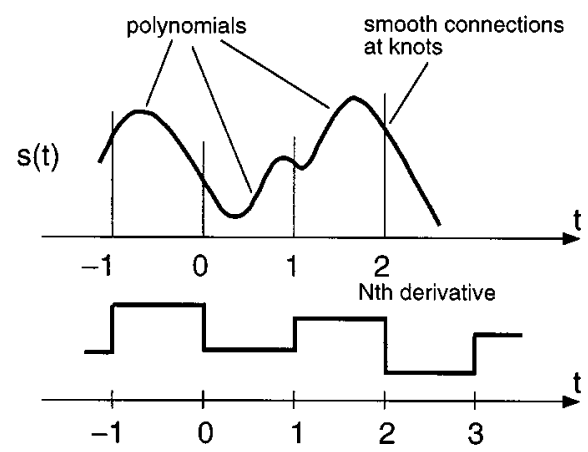

Fig. 7. (a) A spline with knots at integers is a succession of piecewise polynomials, connected at the knots such that it is sufficiently differentiable everywhere. (b) The $N$ th derivative of an $N$ th-order spline is a piecewise constant, typically discontinuous at the knots (integers here).

(a)

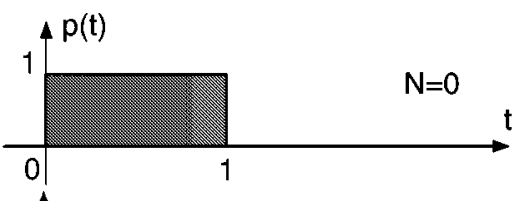

(b)

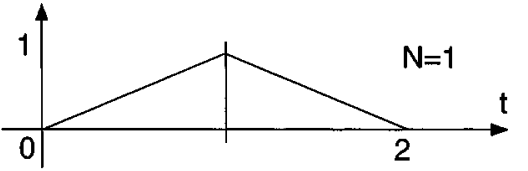

(c)

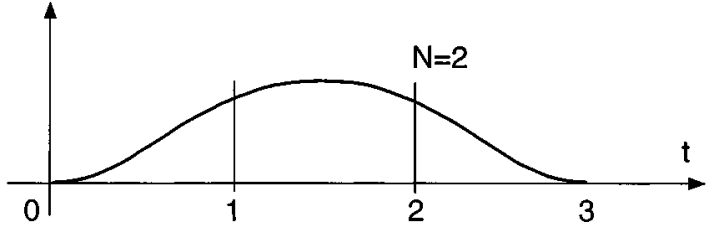

Fig. 8. $N$ th-order $B$-splines or $b_{N}(t)$ for various orders $N$. (a) For $N=0$ this is the pulse, (b) for $N=1$ this is a triangle, and (c) for $N=2$ this is a quadratic. The knots are at integer locations as indicated by vertical lines.

2) At the integers or knots $t=k$, the function $s(t)$ is continuously differentiable $N-1$ times (i.e., differentiable $N-1$ times and the $(N-1)$ th derivative is continuous). $\diamond$

This implies, in fact, that the $N$ th derivative is a piecewise constant with jumps at the knots. Conversely $N$ th-order splines are functions obtained by integrating such piecewise constants $N$ times. Fig. 7 depicts some of the ideas pictorially. There exist splines of finite duration. It can be shown [12] that an $N$ th-order spline has duration at least $N+1$. It is easy to obtain examples of such splines. For this define the pulse function

$$
p(t)= \begin{cases}1, & 0<t<1 \\ 0, & \text { otherwise }\end{cases}
$$

Define the function $b_{N}(t)$ to be the convolution of $p(t)$ with itself $N$ times. It can be shown that this is a spline of order $N$. This is called the of $B$-spline order $N$. Evidently this is nonzero in $0<t<N+1$ (Fig. 8). The Fourier transform of the $N$ th-order $B$-spline is

$$
B_{N}(j \omega)=\exp \left[-j \omega \frac{(N+1)}{2}\right]\left(\frac{\sin \left(\frac{\omega}{2}\right)}{\frac{\omega}{2}}\right)^{N+1} .
$$

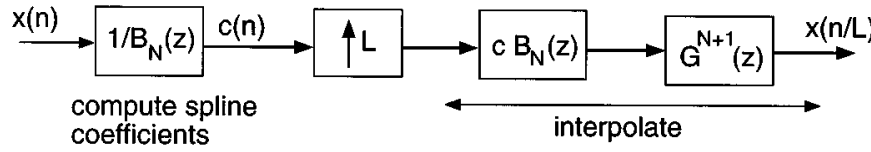

Fig. 9. Interpolation of a signal $x(n)$ with digital filters. The signal is assumed to have a continuous time model $x(t)=\sum_{k} c(k) b_{N}(t-k)$ where $b_{N}(t)$ is the $N$ th-order $B$-spline.

It can be verified that the corresponding time domain expression is

$$
b_{N}(t)=\sum_{k=0}^{N+1}\left(\begin{array}{c}
N+1 \\
k
\end{array}\right)(-1)^{k} \frac{(t-k)^{N}}{N !} \mathcal{U}(t-k)
$$

where $\mathcal{U}(t)$ is the unit-step. The functions $b_{2}(t)$ and $b_{3}(t)$ are known, respectively, as the quadratic and cubic splines. We can verify that

$$
b_{2}(t)= \begin{cases}\frac{t^{2}}{2}, & 0 \leq t<1 \\ \frac{3}{4}-\left(t-\frac{3}{2}\right)^{2}, & 1 \leq t<2 \\ \frac{(t-3)^{2}}{2}, & 2 \leq t<3 \\ 0, & \text { otherwise }\end{cases}
$$

and is shown in Fig. 8(c). $B$-splines are commonly used in interpolation and have been known in the mathematics literature for many decades (e.g., see Schoenberg's classic book [12]). In the digitial signal processing literature, the use of splines specifically for interpolation became practicable because of the important work by Unser et al., [20] who showed that $B$-spline interpolation can be performed efficiently with FIR and stable IIR filters of very low complexity [15]-[17].

\section{B. Cubic Spline Example}

Assuming that $\phi(t)$ is $N$ th-order spline $b_{N}(t)$, we see that the prefilter is the IIR filter $H(z)=1 / B_{N}(z)$ where $B_{N}(z)=$ $\sum_{n=0}^{N+1} b_{N}(n) z^{-n}$. For example if $N=3$ (cubic spline case) we have

$$
B_{3}(z)=\frac{z^{-1}+4 z^{-2}+z^{-3}}{6}
$$

and $H(z)=1 / B_{3}(z)$. Next, the interpolation filter $F(z)$ has impulse response $f(n)=b_{N}(n / L)$. Unser et al. [14] have shown that $F(z)$ can be written in the especially elegant form $F(z)=c B_{N}(z)[G(z)]^{N+1}$ where $c=1 / L^{N}$ and $G(z)$ is the simple running-sum filter

$$
G(z)=\sum_{n=0}^{L-1} z^{-n}=\frac{1-z^{-L}}{1-z^{-1}} .
$$

The implementation of $F(z)$ is therefore computationally very efficient. Fig. 9 shows how Fig. 6 simplifies in this case. The zeros of $B_{3}(z)$ are $z_{1}=-3.7321$ and $z_{2}=-0.2679$ showing that $1 / B_{3}(z)$ is not a causal stable IIR filter. But it can be implemented using a combination of a causal and anticausal stable filter, and the same is true for arbitrary $N$. As emphasized by Unser et al., this is not only a practical scheme for finite length signals (e.g., images), it is also computationally very efficient. A comparison of this approach with several traditional ones can be found in [14]. Fig. 10 shows $512 \times 512$ images obtained by two-fold interpolation $(L=2)$ of a 

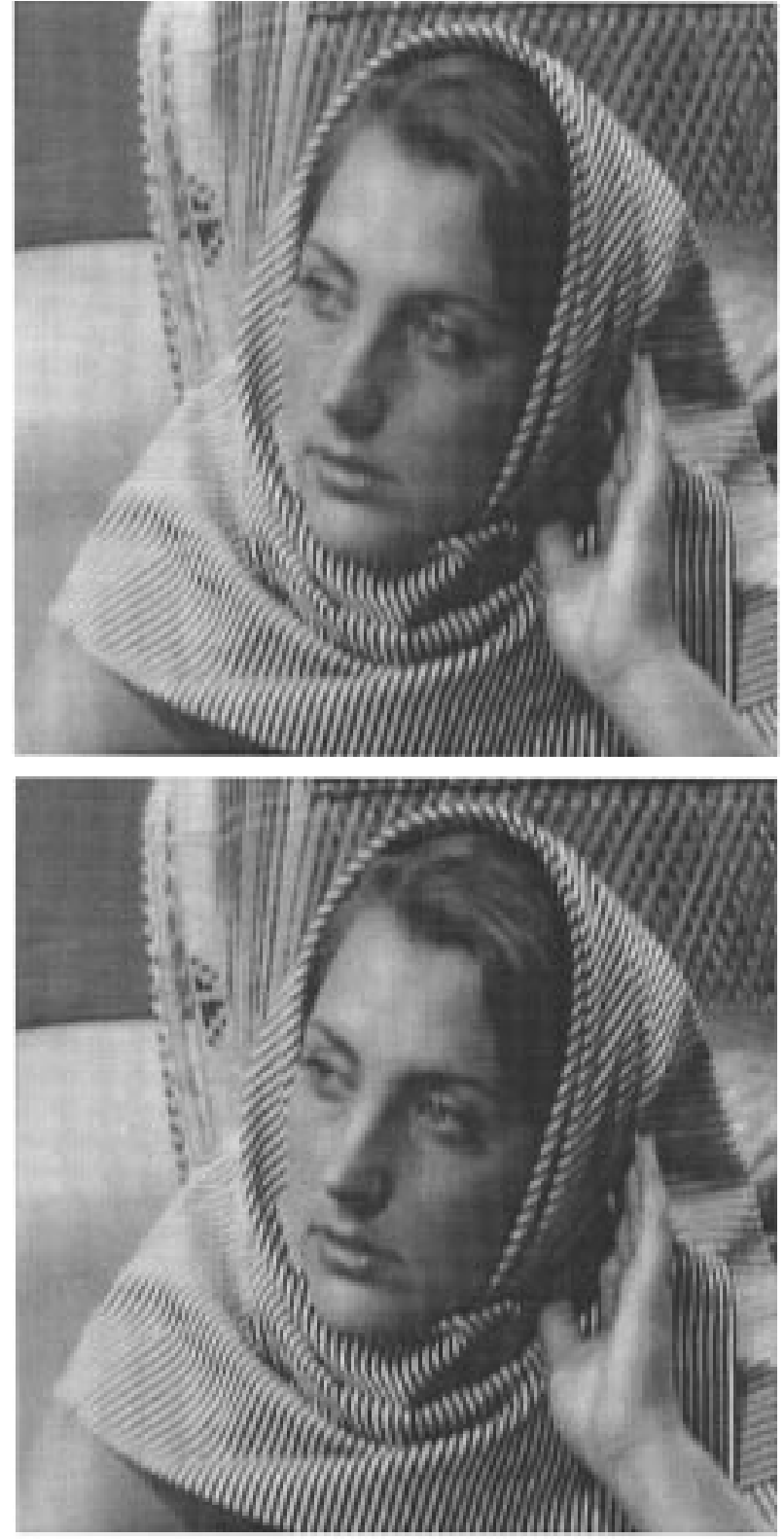

Fig. 10. $512 \times 512$ interpolated versions of a portion of Barbara image using zeroth-order spline (top), and cubic spline (bottom).

$256 \times 256$ section of the Barbara image. Both zeroth-order and cubic-spline interpolations are shown. In the former, the sample and hold effect is very visible and annoying, especially at the locations of the stripes. The cubic spline interpolation is smoother, and the stripes have a much cleaner appearance.

\section{STABiLITY OF RECONSTRUCTION}

Consider the reconstruction formula

$$
x(t)=\sum_{k=-\infty}^{\infty} x(k) s_{k}(t)
$$

Typically, $s_{k}(t)=s(t-k)$, for most cases under discussion. If a slight perturbation of samples $x(k)$ results in a large perturbation of the reconstructed signal as demonstrated in
Fig. 11, then such reconstructions are not useful. Qualitatively speaking, the reconstruction is stable if small errors in the sample values remain small in the reconstructed signal. More precisely, let $x(k)$ be replaced with the noisy version $x(k)+e(k)$. Then the reconstructed signal has the error component $e(t)=\sum_{k=-\infty}^{\infty} e(k) s_{k}(t)$. If we can show that

$$
\int_{-\infty}^{\infty}|e(t)|^{2} d t \leq C \sum_{k=-\infty}^{\infty}|e(k)|^{2}
$$

for some finite constant $C$ independent of $e(k)$, the reconstruction is said to be stable in the energy-sense.

For example, suppose $\left\{s_{k}(t)\right\}$ satisfy orthogonality, that is

$$
\int s_{k}(t) s_{m}^{*}(t) d t=C \delta(k-m)
$$

for some $C>0$. Then, (11) holds with equality assuring this kind of stability. A special case is reconstruction from samples of a bandlimited signal. Here, $s_{k}(t)=\sin \pi(t-k) / \pi(t-k)$ (shifted sine functions) and satisfy the preceding orthogonality property (Appendix I). Reconstruction of a bandlimited signal from its sampled version (sampling rate $\geq$ Nyquist rate) is therefore stable in the energy-sense.

\section{A. Importance of Pointwise Stability}

While stability in the energy sense is nice, a more stringent type of stability called pointwise stability is desirable in the reconstruction of $x(t)$ from samples. Before defining this kind of stability, we first explain why energy-based stability is insufficient. Consider a hypothetical example of a signal $x(t)$ and its noisy reconstructed version $x(t)+e(t)$ shown in Fig. 12. We assume for the purpose of illustration that the error $e(t)$ is in the form of short occassional pulses. Even if the pulse widths in the error $e(t)$ (hence the error energy $\left.\int|| e(t)\right|^{2} d t$ ) are arbitrarily small, the samples of $x(t)+e(t)$ will differ significantly from those of $x(t)$ at certain points. Thus, a resampling of $x(t)+e(t)$ (perhaps with a slight offset of sampling instants) could yield results that are completely different from the original samples of $x(t)$. This situation is avoided by requiring that the reconstruction error be small pointwise in time. For example, if a reconstruction scheme is such that

$$
|e(t)|^{2} \leq C \sum_{k}|e(k)|^{2}
$$

then the preceding mishap will never happen. By making the error in samples small enough we can make the reconstruction error $e(t)$ as small as we want, for all $t$. Whenever (12) holds, we say that the reconstruction from samples is pointwise stable.

\section{B. Pointwise Bound for Bandlimited Signals}

Let $x(n T)$ denote samples of the $\sigma$-BL signal $x(t)$. Assume Nyquist sampling, that is, $T=\pi / \sigma$. The signal $x(t)$ can be reconstructed from $x(n T)$ using the ideal lowpass filter or sine function $h(t)=\sin \sigma t / \sigma t$, according to the equation

$$
x(t)=\sum_{n=-\infty}^{\infty} x(n T) h(t-n T) .
$$




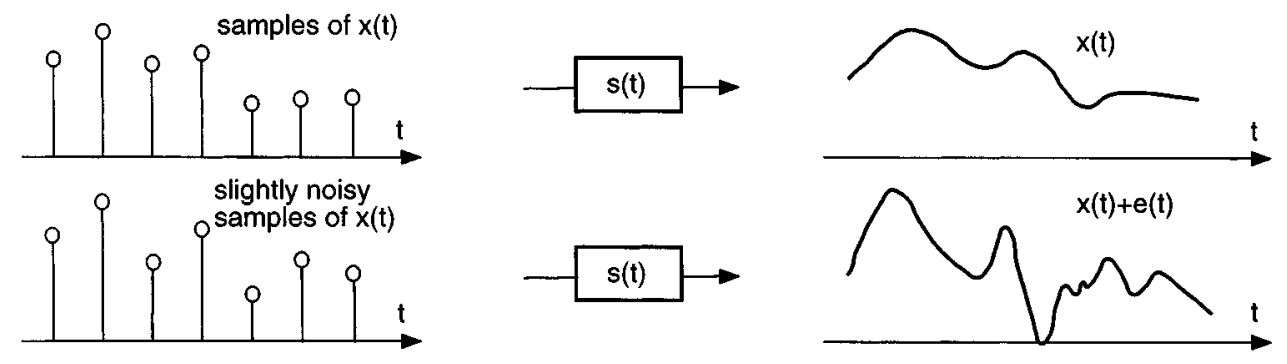

(a)

(b)

Fig. 11. (a) A signal $x(t)$ reconstructed from samples, and (b) a slightly perturbed set of samples resulting in a completely different reconstructed signal $x(t)+$ $e(t)$.

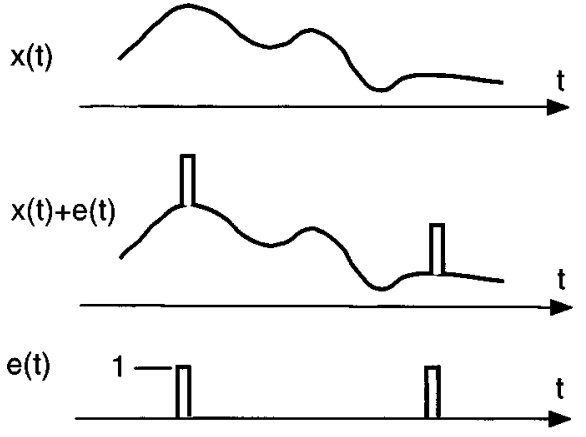

Fig. 12. A signal $x(t)$ and its noisy version $x(t)+e(t)$. The noise $e(t)$ is also shown.

Using Cauchy-Schwartz inequality, it follows that

$$
\left|x\left(t_{0}\right)\right|^{2} \leq \sum_{n=-\infty}^{\infty}|x(n T)|^{2} \sum_{n=-\infty}^{\infty}\left|h\left(t_{0}-n T\right)\right|^{2}
$$

for any $t_{0}$. The sum $\sum_{n}\left|h\left(t_{0}-n T\right)\right|^{2}$ can be regarded as the energy in the samples of the $\sigma$-BL signal $h\left(t_{0}-t\right)$. Applying the energy identity proved in Appendix I for bandlimited signals (32) we therefore have

$$
\begin{aligned}
\sum_{n=-\infty}^{\infty}\left|h\left(t_{0}-n T\right)\right|^{2} & =\frac{1}{T} \int_{-\infty}^{\infty}\left|h\left(t_{0}-t\right)\right|^{2} d t \\
& =\frac{1}{T} \int_{-\infty}^{\infty}|h(t)|^{2} d t \\
& =\sum_{n}|h(n T)|^{2} \text { (energy identity again). } \\
& =1 .
\end{aligned}
$$

The last equality follows from observing that $h(n T)=$ $\sin (\sigma T n) / \sigma T n=\sin \pi n / \pi n=\delta(n)$. Thus, the quantity $t_{0}$ is irrelevant in the summation. Summarizing, we have shown that

$$
|x(t)|^{2} \leq \sum_{n=-\infty}^{\infty}|x(n T)|^{2} \text { for all } t
$$

for any $\sigma$-bandlimited signal sampled at the Nyquist spacing $T=\pi / \sigma$. Applying the energy identity (32), again we see that the preceding also implies

$$
|x(t)|^{2} \leq \frac{1}{T} \int_{-\infty}^{\infty}|x(\tau)|^{2} d \tau \quad \text { for all } t
$$

where $T=\pi / \sigma$. This result makes no reference to sampling at all! It simply says that a bandlimited signal is bounded point-

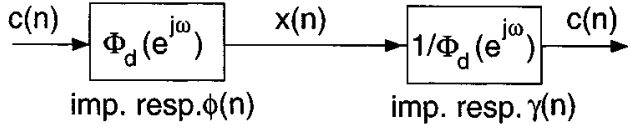

Fig. 13. The digital-filtering relation between $c(n)$ and $x(n)$.

wise in time in terms of its energy. So a fixed amount of energy cannot be concentrated in an arbitrarily narrow region (for that would increase the magnitude arbitrarily somewhere). We now apply this result to study the stability of reconstruction in presence of additive noise in the samples.

Theorem 1. Pointwise Stability in Nyquist Sampling: Let $x(t)$ be a bandlimited signal and let $x(n T)$ represent samples at the Nyquist rate (i.e., $T=\pi / \sigma)$. Let $x(n T)+e(n T)$ represent the samples with error $e(n T)$. Assume the error has finite energy, that is, $\sum_{n=-\infty}^{\infty}|e(n T)|^{2}<\infty$. Then the reconstructed signal is $x(t)+e(t)$ where the error $e(t)$ is bounded pointwise according to $|e(t)|^{2} \leq \sum_{n=-\infty}^{\infty}|e(n T)|^{2}$.

Proof: The error in the reconstructed signal is $e(t)=\sum_{n=-\infty}^{\infty} e(n T) h(t-n T)$. So $e(t)$ is a $\sigma$-BL signal and therefore satisfies $|e(t)|^{2} \leq \sum_{n=-\infty}^{\infty}|e(n T)|^{2}$ for all $t$.

$\nabla \nabla \nabla$

Note that stability of reconstruction is not synonymous with filter stability. The reconstruction filter $s(t)=\sin \sigma t / \sigma t$ is unstable in the BIBO sense [10]. Still the reconstruction process is pointwise stable.

\section{Pointwise Stability in MR Sampling}

Consider again signals of the form $x(t)=\sum_{k} c(k) \phi(t-k)$ discussed in Section II. We will make a careful choice of mathematical assumptions and establish pointwise stability of the reconstruction equation $x(t)=\sum_{k} x(k) s(t-k)$. The samples of this signal, taken at integer spacing, are given by

$$
x(n)=\sum_{k} c(k) \phi(n-k) .
$$

Assuming that $\Phi_{d}\left(e^{j \omega}\right) \triangleq \sum_{n} \phi(n) e^{-j \omega n} \neq 0$ for all $\omega$, we can construct $c(n)$ from the samples $x(n)$ using

$$
c(n)=\sum_{k} x(k) \gamma(n-k)
$$

where $\gamma(n)$ is the impulse response of $1 / \Phi_{d}\left(e^{j \omega}\right)$. Equations (14) and (15) represent two digital filters which are convolutional inverses of each other (see Fig. 13).

Our first goal is to ensure that both of these filters are BIBO stable [10], that is, the impulse responses are $\ell_{1}$ sequences (i.e., 
absolutely summable). For this we make the assumption that $\phi(t)$ is bounded as

$$
|\phi(t)| \leq \frac{K}{1+|t|^{1+\epsilon}}
$$

for some $K>0$ and $\epsilon>0$ (in words, $\phi(t)$ decays faster than $1 / t$ ). Under this and a few other mild assumptions we will establish stability properties. The following presentation differs in style as well as detail from some of the original results of Walter [29] who first studied this problem rigorously.

Lemma 1. Filter Properties: Suppose $\phi(t)$ is bounded as in (16) and furthermore $\Phi_{d}\left(e^{j \omega}\right) \triangleq \sum_{n} \phi(n) e^{-j \omega n} \neq 0$ for all $\omega$. Then (a) $\phi(n)$ and $\gamma(n)$ are $\ell_{1}$ sequences, (b) $\phi(n)$ and $\gamma(n)$ are also $\ell_{2}$ sequences, and (c) the Fourier transforms of $\phi(n)$ and $\gamma(n)$ are finite for all $\omega$.

Proof: Equation (16) means that $|\phi(n)|<K /|n|^{1+\epsilon}, n \neq$ 0 , which assures that $\phi(n) \in \ell_{1}$. Now there is a theorem due to Wiener [11] which says that if $\phi(n) \in \ell_{1}$ and $\Phi_{d}\left(e^{j \omega}\right) \neq 0$ for all $\omega$ then $\gamma(n)$ is also an $\ell_{1}$ signal. Next, if $\phi(n)$ is an $\ell_{1}$ sequence then it is also an $\ell_{2}$ sequence because $\sum_{n}|\phi(n)|<\infty$ implies $|\phi(n)|<\alpha$ for all $n$ some finite $\alpha$ so that $\sum_{n}|\phi(n)|^{2} \leq$ $\alpha \sum_{n}|\phi(n)|<\infty$. The $\ell_{1}$ property also implies that the Fourier transform is finite because $\left|\Phi_{d}\left(e^{j \omega}\right)\right| \leq \sum_{n}\left|\phi(n) e^{-j \omega n}\right|=$ $\sum_{n}|\phi(n)|<\infty$.

$\nabla \nabla \nabla$

Lemma 2: Suppose $\phi(t)$ is bounded as in (16) and furthermore $\Phi_{d}\left(e^{j \omega}\right) \triangleq \sum_{n} \phi(n) e^{-j \omega n} \neq 0$ for all $\omega$. Assume further that $c(n)$ is in $\ell_{2}$. Then $x(n)$ is in $\ell_{2}$ and furthermore there are finite positive constants $A, B$ such that $A \sum_{n}|x(n)|^{2} \leq$ $\sum_{n}|c(n)|^{2} \leq B \sum_{n}|x(n)|^{2}$.

Proof: This is essentially a consequence of the facts that (a) $x(n)$ is the output of a BIBO stable filter with input $c(n)$, and (b) the inverse of this filter is also BIBO stable. For a formal proof we will use the fact that $\left|\Phi_{d}\left(e^{j \omega}\right)\right| \leq \sum_{n}\left|\phi(n) e^{-j \omega n}\right|=$ $\sum_{n}|\phi(n)|<\infty$ because $\phi(n) \in \ell_{1}$. Thus

$$
\begin{aligned}
\sum_{n}|x(n)|^{2} & =\int_{0}^{2 \pi} \frac{1}{2 \pi}\left|X\left(e^{j \omega}\right)\right|^{2} d \omega \\
& =\int_{0}^{2 \pi} \frac{1}{2 \pi}\left|C\left(e^{j \omega}\right) \Phi_{d}\left(e^{j \omega}\right)\right|^{2} d \omega \\
& \leq \frac{1}{A} \int_{0}^{2 \pi} \frac{1}{2 \pi}\left|C\left(e^{j \omega}\right)\right|^{2} d \omega=\frac{1}{A} \sum_{n}|c(n)|^{2}
\end{aligned}
$$

where $1 / A=\left(\sum_{n}|\phi(n)|\right)^{2}$. This shows in particular that $x(n)$ is also an $\ell_{2}$ sequence. Next, since $c(n)$ is the output of the filter $\gamma(n)$ in response to $x(n)$, we similarly have $\sum_{n}|c(n)|^{2} \leq$ $B \sum_{n}|x(n)|^{2}$ where $B=\left(\sum_{n}|\gamma(n)|\right)^{2}<\infty$ because $\gamma(n) \bar{\epsilon}$ $\ell_{1}$.

$\nabla \nabla \nabla$

Theorem 2. Pointwise Bound in MR Sampling: Let $x(t)=$ $\sum_{n} c(n) \phi(t-n)$ where $\phi(t)$ is bounded as in (16) and furthermore $\Phi_{d}\left(e^{j \omega}\right) \triangleq \sum_{n} \phi(n) e^{-j \omega n} \neq 0$ for all $\omega$. Assume further that $c(n)$ is in $\ell_{2}$. Then

$$
|x(t)|^{2} \leq \alpha \sum_{n}|x(n)|^{2} \quad \text { for all } t
$$

for some finite nonzero constant $\alpha$.
Proof: Applying Cauchy-Schwartz inequality, the equation $x\left(t_{0}\right)=\sum_{n} c(n) \phi\left(t_{0}-n\right)$ yields

$$
\left|x\left(t_{0}\right)\right|^{2} \leq \sum_{n}|c(n)|^{2} \sum_{n}\left|\phi\left(t_{0}-n\right)\right|^{2} .
$$

To proceed further note that $\phi\left(t_{0}-n\right)$ represents samples of the shifted function $\phi\left(t_{0}-t\right)$. If $\phi(t)$ is bounded as in (16) then so is $\phi\left(t_{0}-t\right)$ for appropriate $K$ and $\epsilon$ in (16). So $\phi\left(t_{0}-n\right)$ is in $\ell_{2}$ (Lemma 1) so that $\sum_{n}\left|\phi\left(t_{0}-n\right)\right|^{2}$ in the preceding equation is finite. Next, from Lemma 2 we see that $\sum_{n}|c(n)|^{2} \leq$ $B \sum_{n}|x(n)|^{2}$ for a finite positive $B$. Thus

$$
\left|x\left(t_{0}\right)\right|^{2} \leq \sum_{n}|c(n)|^{2} \sum_{n}\left|\phi\left(t_{0}-n\right)\right|^{2} \leq \alpha \sum_{n}|x(n)|^{2}
$$

for some finite positive $\alpha$.

$\nabla \nabla \nabla$

Corollary 1. Pointwise Stability: The preceding theorem immediately implies pointwise stability of reconstruction. Thus let $x(n)+e(n)$ represent the samples with error $e(n)$ and assume $\sum_{n=-\infty}^{\infty}|e(n)|^{2}<\infty$. Then we can use the stable filter $1 / \Phi_{d}\left(e^{j \omega}\right)$ to define an $\ell_{2}$ sequence $c_{e}(n)$ such that $e(n)=$ $\sum_{k} c_{e}(k) \phi(n-k)$. The signal $e(t) \triangleq \sum_{k} c_{e}(k) \phi(t-k)$ is then the additive noise affecting $x(t)$. Applying (17) to $e(t)$ we find

$$
|e(t)|^{2} \leq \alpha \sum_{n}|e(n)|^{2}
$$

which is precisely the pointwise stability property. We conclude this section with a technical detail.

Theorem 3. Pointwise Bound and Riesz Bias: Let all assumptions be as in Theorem 2. Assume further that $\{\phi(t-k)\}$ is a Riesz basis for signals of the form $x(t)=\sum_{k} c(k) \phi(t-k)$ where $c(k) \in \ell_{2}$. Then in addition to (17) we also have

$$
|x(t)|^{2} \leq \beta \int_{-\infty}^{\infty}|x(\tau)|^{2} d \tau \text { for all } t
$$

for some finite nonzero constant $\beta$. Thus, similar to bandlimited signals, $x(t)$ is bounded pointwise in time in terms of its energy. $\diamond$

Proof: By definition $\{\phi(t-k)\}$ is said to be a Riesz basis if there exists finite nonzero constants $A_{1}$ and $B_{1}$ such that for any $c(n) \in \ell_{2}$

$$
A_{1} \sum_{n}|c(n)|^{2} \leq \int_{-\infty}^{\infty}|x(\tau)|^{2} d \tau \leq B_{1} \sum_{n}|c(n)|^{2}
$$

where $x(\tau)=\sum_{n} c(n) \phi(\tau-n)$. We will need only the first inequality in the proof. Application of Cauchy-Schwartz inequality in $x(t)=\sum_{n} c(n) \phi(t-n)$ yields

$$
\begin{aligned}
\left|x\left(t_{0}\right)\right|^{2} & \leq \sum_{n}\left|\phi\left(t_{0}-n\right)\right|^{2} \sum_{n}|c(n)|^{2} \\
& \leq \sum_{n}\left|\phi\left(t_{0}-n\right)\right|^{2} \int_{-\infty}^{\infty}|x(\tau)|^{2} \frac{d \tau}{A_{1}} \\
& =\beta \int_{-\infty}^{\infty}|x(\tau)|^{2} d \tau
\end{aligned}
$$

where $\beta=\sum_{n}\left|\phi\left(t_{0}-n\right)\right|^{2} / A_{1}$. 


\section{Pointwise Stability and Functional Subspaces}

The stablility of reconstruction of $x(t)$ from samples is related to the idea of a functional space. Recall that $L_{2}$ denotes the Hilbert space [7] of functions with finite energy $\|x(t)\|^{2} \triangleq$ $\int|x(t)|^{2} d t$. Let $\mathcal{H}$ be a subspace with the additional property that $|x(t)|$ is pointwise bounded as follows:

$$
|x(t)|^{2} \leq C \int_{-\infty}^{\infty}|x(\tau)|^{2} d \tau
$$

where $C<\infty$. The constant $C$ is the same for all $x(t)$ in $\mathcal{H}$ though it might depend ${ }^{2}$ on the choice of the subspace $\mathcal{H}$. A subspace satisfying the preceding inequality is called a functional space or a reproducing kernel Hilbert space (rkhs). Notice that arbitrary subspaces of $L_{2}$ may not satisfy (20) (e.g., $\mathcal{H}=L_{2}$ ) because we can change the value of $x(t)$ at a point $t_{0}$ by an arbitrary amount without changing $\int|x(\tau)|^{2} d \tau$.

We proved that the subspace of $\sigma$-BL signals satisfies (13) and is therefore a functional subspace. Similarly Theorem 3 shows that the subspace of signals of the form $x(t)=\sum_{n} c(n) \phi(t-n)$ is a functional subspace under the assumptions mentioned in that theorem. So, we have seen two explicit examples of function subspaces. In such spaces pointwise stability of reconstruction from samples is guaranteed (i.e., we get "good" sampling theorems). For further reading on this topic, the reader is referred to [2] and [19].

\section{NONUNIFORM SAMPLING}

If $\phi(t)$ is compactly supported (e.g., Daubechies' scaling function, or a member of the spline family, etc.) then the sequence $\phi(n)$ is an FIR filter and its inverse $1 / \Phi\left(e^{j \omega}\right)$ is IIR. So the construction of $c(n)$ (hence $x(t)$ ) from $x(n)$ involves IIR filtering (review Fig. 13). Mallat [8] proposed a clever way to avoid such filtering by oversampling $x(t)$ at a much higher resolution. The idea is that if $x(t)$ belongs to $V_{0}$ it also belongs to finer spaces such as $V_{N}, N>0$. This is called the multiresolution property, and is ensured by further restrictions on $\phi(t)$. Thus

$$
x(t)=\sum_{k} a(k) 2^{N / 2} \phi\left(2^{N} t-k\right) .
$$

If $N$ is large enough then $2^{N / 2} \phi\left(2^{N} t-k\right)$ are narrow enough to make the approximation $a(k) \approx \alpha x\left(2^{-N} k\right)$ for some constant $\alpha$. The coefficients $c(k)$ can then be found from $a(k)$ by using a discrete time filter bank (see [25] and [28] for tutorial review). It turns out that such arbitrary oversampling is not necessary; oversampling by a factor of two is enough. Under mild conditions $c(k)$ can be computed exactly from $x(0.5 n)$ with the help of FIR filters alone [28].

In fact both IIR filtering and oversampling schemes can be avoided completely if the samples of $x(t)$ are allowed to be nonuniform. Thus, we will show how to reconstruct $c(n)$ from periodically nonuniform samples of $x(t)$ with the help of FIR filters alone. For example, suppose we consider the following three sets of samples:

$$
x(3 n) \quad x\left(3 n+\frac{1}{3}\right) \quad x\left(3 n+\frac{2}{3}\right) .
$$

${ }^{2}$ Actually $C$ could depend on $t$ but we keep it simple here.

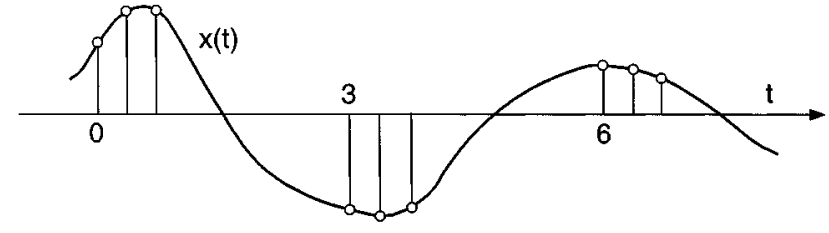

Fig. 14. Demonstration of nonuniform sampling.

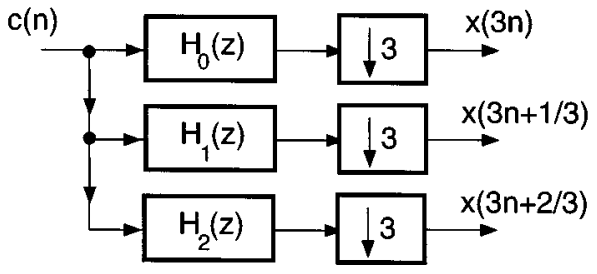

Fig. 15. Analysis bank representing nonuniform sampling.

This is equivalent to the nonuniform sampling scheme shown in Fig. 14, with average rate still equal to unity. The three sets of samples can be expressed as

$$
x\left(3 n+\frac{i}{3}\right)=\sum_{k} c(k) \phi\left(3 n+\frac{i}{3}-k\right), \quad i=0,1,2 .
$$

We cannot interpret this as convolution as we did in the uniform case. Define the three discrete time filters $H_{i}(z)$ with impulse resposes

$$
h_{i}(n)=\phi\left(n+\frac{i}{3}\right), \quad i=0,1,2 .
$$

That is, $h_{i}(n)$ is the sampled version of a shifted version of $\phi(t)$. Thus we can represent the set of nonuniform samples as the decimated subband signals of a maximally decimated analysis bank (Fig. 15). If there exists an FIR synthesis filter bank with the perfect reconstruction property [23] then we can reconstruct $c(n)$ from these nonuniform samples. Then $x(t)$ can be reconstructed from $x(t)=\sum_{n} c(n) \phi(t-n)$. Most readers familiar with filter bank theory will realize that it is very easy to find examples where such FIR synthesis filters do exist. We now supply a detailed example where $\phi(t)$ is the quadratic spline given in (9). The three analysis filters obtained by sampling this spline are given by

$$
\begin{aligned}
& H_{0}(z)=\frac{z^{-1}+z^{-2}}{2} \quad H_{1}(z)=\frac{1+13 z^{-1}+4 z^{-2}}{18} \\
& H_{2}(z)=\frac{4+13 z^{-1}+z^{-2}}{18} .
\end{aligned}
$$

The $3 \times 3$ polyphase matrix $\mathbf{E}(z)$ of this anaylsis bank [23] is nonsingular and can be inverted to obtain the synthesis bank polyphase matrix $\mathbf{R}(z)$

$$
\begin{aligned}
\mathbf{E}(z) & =\frac{1}{18}\left[\begin{array}{ccc}
0 & 9 & 9 \\
1 & 13 & 4 \\
4 & 13 & 1
\end{array}\right] \\
\mathbf{R}(z) & =\mathbf{E}^{-1}(z)=\frac{1}{4}\left[\begin{array}{ccc}
13 & -36 & 27 \\
-5 & 12 & -3 \\
13 & -12 & 3
\end{array}\right]
\end{aligned}
$$




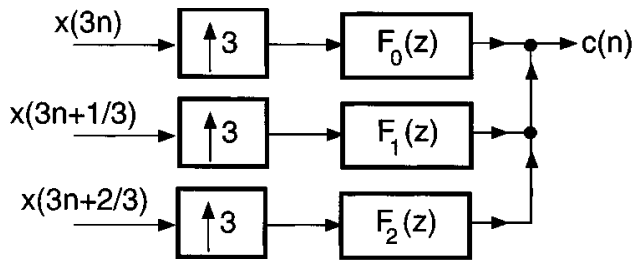

Fig. 16. FIR synthesis bank for reconstructing $c(n)$.

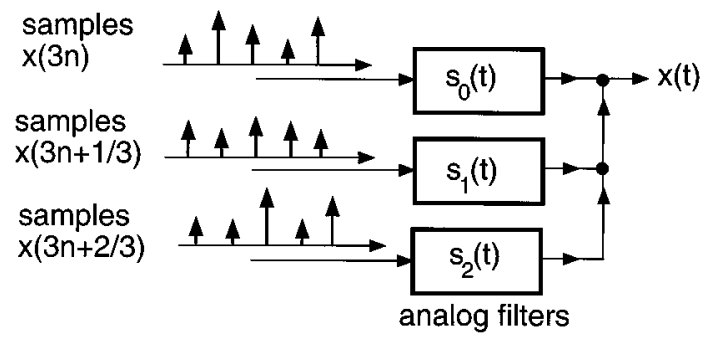

Fig. 17. Reconstruction of $x(t)$ from nonuniform samples.

which shows that the synthesis filters for perfect reconstruction are the FIR filters

$$
\begin{aligned}
& F_{0}(z)=\frac{13-5 z+13 z^{2}}{4} \quad F_{1}(z)=\frac{-36+12 z-12 z^{2}}{4} \\
& F_{2}(z)=\frac{27-3 z+3 z^{2}}{4} .
\end{aligned}
$$

Fig. 16 shows the synthesis bank which reconstructs $c(n)$ from the nonuniform samples of $x(t)$ perfectly. By using $x(t)=$ $\sum_{k} c(k) \phi(t-k)$ we can directly express $x(t)$ in terms of the nonuniform samples:

$$
x(t)=\sum_{i=0}^{2} \sum_{k=-\infty}^{\infty} x\left(3 k+\frac{i}{3}\right) s_{i}(t-3 k) .
$$

Here, $s_{i}(t)$ are related to the scaling function $\phi(t)$ and the synthesis filters by $s_{i}(t)=\sum_{k} f_{i}(k) \phi(t-k)$. We can interpret this reconstruction as a continuous-time filter bank as shown in Fig. 17. Thus, the three sets of samples are passed through three analog filters $s_{0}(t), s_{1}(t)$ and $s_{2}(t)$ and then added up to obtain $x(t)$. Since $f_{i}(k)$ have finite durations, the filters $s_{i}(t)$ also have finite durations like $\phi(t)$.

\section{DisCRETE Time SAMPLING THEOREMS}

A discrete-time signal $x(n)$ is said to be $\sigma$-bandlimited if $X\left(e^{j \omega}\right)=0$ for $\sigma \leq|\omega|<\pi$. If $\sigma=\pi / M$ for integer $M$, we can reconstruct $x(n)$ from the decimated or subsampled version $x(M n)$ [23]. Similar statements can be made with suitably defined bandpass signals. If $x(n)$ is not bandlimited at all (e.g., $X\left(e^{j \omega}\right)$ nonzero everywhere), can we still reconstruct $x(n)$ from a decimated version? Consider Fig. 18 which shows an interpolation filter. The output is given by $x(n)=$ $\sum_{k} f(n-M k) y(k)$.

If the filter $F(z)$ has the Nyquist $(M)$ property

$$
f(M n)=\delta(n)
$$

then we can see that the samples $y(n)$ are also present in the sequence $x(n)$. More precisely we have $y(0)=x(0), y(1)=$ $x(M)$, and so forth, that is, $y(n)=x(M n)$. This shows that

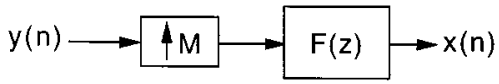

Fig. 18. Signal model allowing recovery from samples.

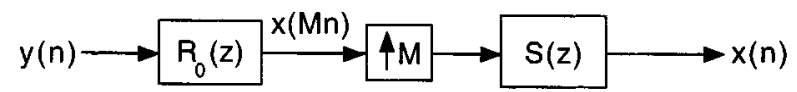

Fig. 19. Extracting the reconstruction filter $S(z)$.

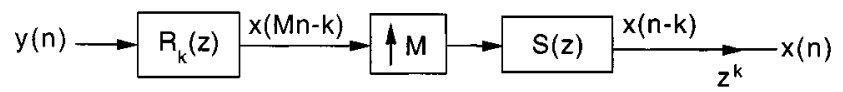

Fig. 20. Extracting the stable reconstruction filter $S(z)$ when $R_{k}(z)$ has no unit circle zeros.

$x(n)$ can be reconstructed perfectly from its decimated version $x(M n)$ even though $x(n)$ may not be bandlimited (because $F(z)$ is not necessarily an ideal bandlimiter in this discussion). The only condition on $F(z)$ is the Nyquist $(M)$ condition. To see how this condition can be eliminated, consider the polyphase representation [23]

$$
F(z)=\sum_{i=0}^{M-1} z^{i} R_{i}\left(z^{M}\right)
$$

$F(z)$ is Nyquist $(M)$ if and only if the 0 th polyphase component $R_{0}(z)=1$. If this is not the case, rewrite

$$
F(z)=R_{0}\left(z^{M}\right) \underbrace{\left(1+z S_{1}\left(z^{M}\right)+\cdots+z^{M-1} S_{M-1}\left(z^{M}\right)\right)}_{\text {call this } S(z)}
$$

so that the filter $S(z)$ is Nyquist $(M)$. Using a standard multirate identity, we can then redraw Fig. 18 as in Fig. 19. This shows that $x(n)$ can still be recovered from $x(M n)$ if we use the interpolation filter

$$
S(z) \triangleq \frac{F(z)}{R_{0}\left(z^{M}\right)} .
$$

We call $F(z)$ the model filter (it defines the signal model in Fig. 18), and $S(z)$ the reconstruction filter.

\section{A. Stability of Reconstruction}

In order to be practical, the reconstruction filter $S(z)$ has to be stable and preferrably causal. Assume the model filter $F(z)$ is stable. If $R_{0}(z)$ has all zeros inside the unit circle then $S(z)=F(z) / R_{0}\left(z^{M}\right)$ is a causal stable IIR filter. In fact if $R_{0}(z)$ has no unit-circle zeros, $S(z)$ has a (possibly noncausal) stable impulse response, and this is in principle sufficient to get stable reconstruction. More generally, if the $k$ th-polyphase component $R_{k}(z)$ has no unit circle zeros, we can rewrite $F(z)=z^{k} R_{k}\left(z^{M}\right) S(z)$ where $S(z)$ is $\operatorname{Nyquist}(M)$. The signal model can therefore be redrawn as in Fig. 20. We see that $x(n-k)$ (hence $x(n))$ can be recovered from its decimated version $x(M n-k)$ using the stable IIR filter

$$
S(z)=\frac{z^{-k} F(z)}{R_{k}\left(z^{M}\right)} .
$$

The preceding discussion also shows that stable reconstructibility from the decimated version of $x(n-k)$ (i.e., from $x(M n-k)$ ) for some $k$ does not imply the same from 


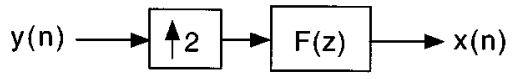

(b)

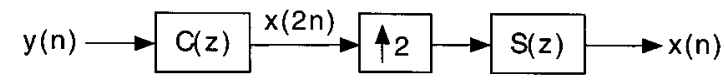

Fig. 21. (a) The signal model. (b) Simplification in the case of Example 2.

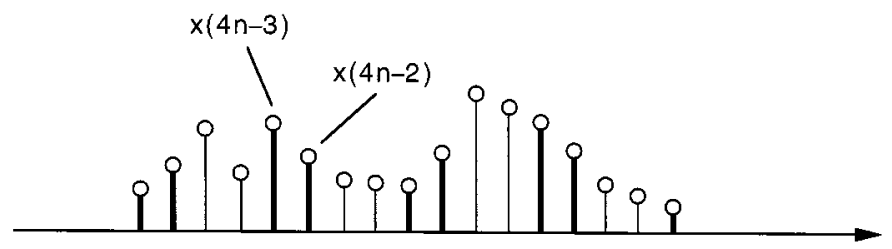

Fig. 22. Demonstrating nonuniform decimation.

$x(M n-i)$ for $i \neq k$. Notice finally that if all the polyphase components have unit-circle zeros then we cannot use the preceding trick. A totally different technique is needed based on nonuniform decimation (Section VI-C).

Example 1. Unit Circle Zeros: Let the model filter be $F(z)=1+z-z^{2}+z^{3}$. Assuming $M=2$ we have $R_{0}(z)=1-z$ and $R_{1}(z)=1+z$. Both of these polyphase components have unit circle zeros. We cannot therefore reconstruct $x(n)$ from either $x(2 n)$ or $x(2 n-1)$ in a stable manner.

Example 2. Common Factors: Let $F(z)=1+z+z^{2}+z^{3}$. Assuming $M=2$ we have $R_{0}(z)=R_{1}(z)=1+z$. Both of these polyphase components have unit circle zeros. In this example we can rewrite $F(z)=C\left(z^{2}\right)(1+z)$ where $C(z)=1+z$ is the common factor between the polyphase components. The signal model can therefore be simplified as shown in Fig. 21 using a standard multirate identity [23]. This shows that we can reconstruct $x(n)$ from $x(2 n)$ by using the Nyquist(2) filter $S(z)=1+z$. This example demonstrates the fact that if there is a common factor $C(z)$ among the $M$ polyphase components, it can be eliminated altogether from any discussion of reconstructibility.

In the case of Example 1, we will show that it is possible to obtain stable reconstruction if a nonuniformly decimated version of $x(n)$ is used instead of using $x(2 n)$ or $x(2 n-1)$. To explain what this means consider the pair of signals $x(2 M n-i)$ and $x(2 M n-i-1)$ for some fixed $i$. These together constitute a nonuniformly decimated version. The decimation ratio is $M$ because we retain one out of $M$ samples on the average. This is demonstrated in Fig. 22 for $M=2$ and $i=2$. The samples shown in bold lines constitute the nonuniformly decimated version. More generally let $L$ be an arbitrary integer and consider the signals

$$
x_{i}(n)=x(M L n-i), \quad 0 \leq i \leq M L-1 .
$$

Thus $x_{i}(n)$ is a polyphase component of $x(n)$ with respect to the integer $M L$, that is, $X(z)=\sum_{i=0}^{M L-1} z^{i} X_{i}\left(z^{M L}\right)$. Suppose we retain a subset of $L$ signals

$$
x_{i_{0}}(n), x_{i_{1}}(n), \ldots x_{i_{L-1}}(n)
$$

and discard the rest. This set of $L$ signals constitutes a nonuniformly decimated version of $x(n)$. Since we retain $L$ out of $L M$ components, the decimation ratio is $M$. Since the integer $L$ is

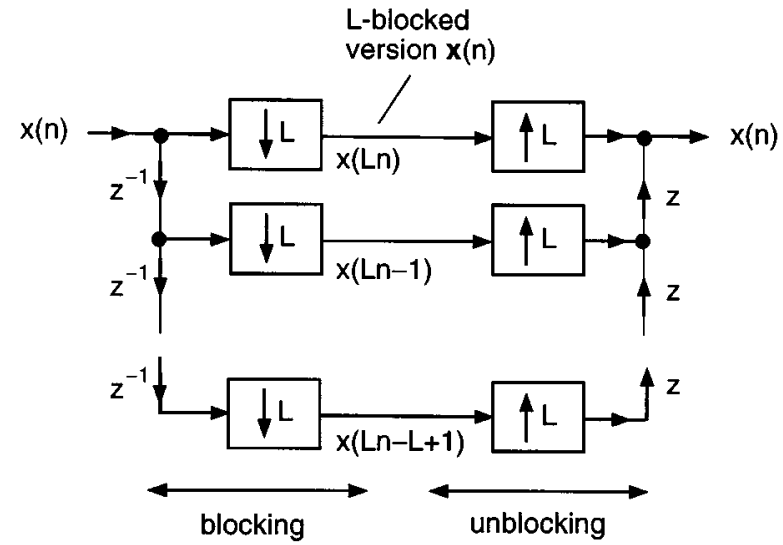

Fig. 23. Blocked and unblocking a signal $x(n)$.

arbitrary (independent of $M$ ) it adds flexibility to the class of nonuniform decimators that can be created this way.

\section{B. Block Representation}

To explain how nonuniform decimation helps in stable reconstruction we first review a definition. Given a signal $x(n)$, its $L$-blocked version is the $L$-component vector

$$
\mathbf{x}(n)=\left[\begin{array}{c}
x(L n) \\
x(\operatorname{Ln}-1) \\
\vdots \\
x(L n-L+1)
\end{array}\right] .
$$

We also say that $x(n)$ is the unblocked version of $\mathrm{x}(n)$. The components $x(L n-i)$ are nothing but the polyphase components of $x(n)$ with respect to $L$. The integer $L$ is known as the block length. Fig. 23 shows how blocking and unblocking can be represented using multirate building blocks.

Now, consider a transfer function $H(z)$ with input $x(n)$ and output $y(n)$ [Fig. 24(a)]. Redraw this as in Fig. 24(b) where $\mathbf{x}(n)$ and $\mathbf{y}(n)$ are $L$-blocked versions of $x(n)$ and $y(n)$. Then, the mapping from $\mathbf{x}(n)$ and $\mathbf{y}(n)$ is a linear time invariant system [23] with an $L \times L$ transfer matrix $\mathbf{H}(z)$ called the blocked version of $H(z)$. Letting $H(z)=\sum_{k=0}^{L-1} z^{-k} E_{k}\left(z^{L}\right)$ be the Type 1 polyphase form of $H(z)$ (Section I-B), the blocked version for $L=3$ has the form

$$
\mathbf{H}(z)=\left[\begin{array}{ccc}
E_{0}(z) & E_{1}(z) & E_{2}(z) \\
z^{-1} E_{2}(z) & E_{0}(z) & E_{1}(z) \\
z^{-1} E_{1}(z) & z^{-1} E_{2}(z) & E_{0}(z)
\end{array}\right] .
$$

In this matrix, any row is obtained from the previous row by shifting it to the right and then recirculating the last element that spills over. In this sense it is similar to a circulant matrix, but since the recirculated element is also multiplied by $z^{-1}$, it is formally called a pseudocirculant matrix [21], [23].

\section{Reconstruction from Nonuniform Decimation}

The signal model for $x(n)$, given by Fig. 18 can be redrawn in polyphase form as shown in Fig. 25, where $F(z)=\sum_{k=0}^{M-1} z^{k} R_{k}\left(z^{M}\right)$ as usual. Now imagine that each polyphase component $R_{k}(z)$ is represented in $L$-blocked form for some $L$ as in Fig. 24. By combining the expander $\uparrow L$ in this system with the expander $\uparrow M$ in Fig. 25 we can redraw Fig. 25 


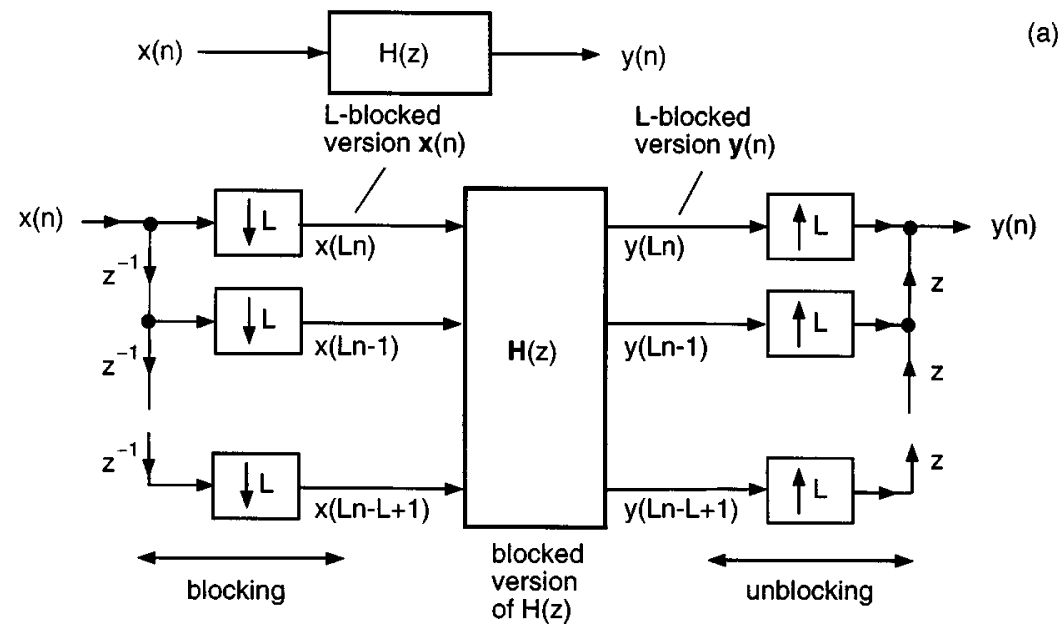

(b)

Fig. 24. (a) A transfer function $H(z)$, and (b) its $L$-blocked version $\mathbf{H}(z)$.

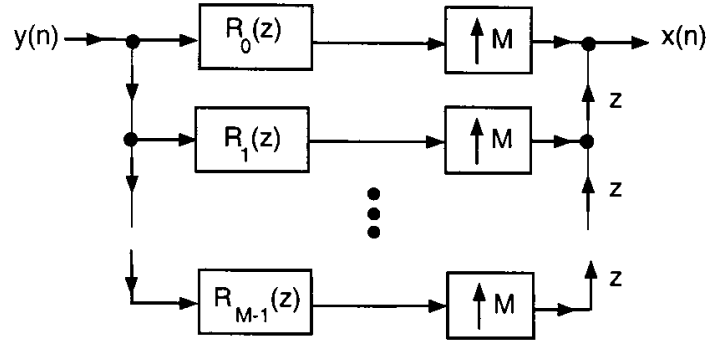

Fig. 25. The standard polyphase representation of the signal model of Fig. 18.

as shown in Fig. 26. The advance operators $z$ in Fig. 24 become $z^{M}$ because they are moved to the right of the expanders $\uparrow M$ using noble identities [23].

The signals $x_{i}(n)$ appearing in Fig. 26 are the $M L$ polyphase components of $x(n)$ defined in (26). Consider a subset $x_{i_{0}}(n), x_{i_{1}}(n), \ldots x_{i_{L-1}}(n)$. This subset defines a nonuniformly decimated version of $x(n)$. It can be used to determine $x(n)$ if the driving signal $y(n)$ in the model Fig. 18 can be determined. This in turn is possible if and only if the $L$ signals $y_{k}(n)$ in Fig. 25 can be reconstructed from the above $L$ signals $x_{i}(n)$. To explore this let us formulate the problem in matrix notation. Let $\mathrm{x}_{k}(n)$ denote the vector of $L$ outputs of $\mathbf{R}_{k}(z)$ and $\mathbf{X}_{k}(z)$ its $z$-transform. We can then write

$$
\left[\begin{array}{c}
\mathbf{X}_{0}(z) \\
\mathbf{X}_{1}(z) \\
\vdots \\
\mathbf{X}_{M-1}(z)
\end{array}\right]=\underbrace{\left[\begin{array}{c}
\mathbf{R}_{0}(z) \\
\mathbf{R}_{1}(z) \\
\vdots \\
\mathbf{R}_{M-1}(z)
\end{array}\right]}_{\mathbf{R}_{\text {big }}(z)}\left[\begin{array}{c}
Y_{0}(z) \\
Y_{1}(z) \\
\vdots \\
Y_{L-1}(z)
\end{array}\right] .
$$

Here, $\mathbf{R}_{\text {big }}(z)$ is an $M L \times L$ matrix. It has the $L$ inputs $y_{k}(n)$, $0 \leq k \leq L-1$ and $M L$ outputs $x_{i}(n)$. If the $L$ inputs of $\mathbf{R}_{\text {big }}(z)$ can be reconstructed from a subset of $L$ outputs

$$
x_{i_{0}}(n), x_{i_{1}}(n), \ldots x_{i_{L-1}}(n)
$$

we say that $y(n)$, and hence $x(n)$, has been reconstructed from the $M$-fold nonuniformly decimated version (27). We can write

$$
\left[\begin{array}{c}
X_{i_{0}}(z) \\
X_{i_{1}}(z) \\
\vdots \\
X_{i_{L-1}}(z)
\end{array}\right]=\mathbf{R}_{\mathrm{sub}}(z)\left[\begin{array}{c}
Y_{0}(z) \\
Y_{1}(z) \\
\vdots \\
Y_{L-1}(z)
\end{array}\right]
$$

for an appropriately defined $L \times L$ submatrix $\mathbf{R}_{\text {sub }}(z)$ of $\mathbf{R}_{\text {big }}(z)$. Thus, as long as there exists such a submatrix with $\left[\operatorname{det} \mathbf{R}_{\text {sub }}\left(e^{j \omega}\right)\right] \neq 0$ for any $\omega$, we can obtain the inverse $\mathbf{R}_{\text {sub }}^{-1}\left(e^{j \omega}\right)$, and in principle reconstruct $x(n)$ from the decimated version. For the case where $F(z)$ is an FIR filter, $\mathbf{R}_{\text {sub }}(z)$ is a polynomial matrix and the condition is that [det $\mathbf{R}_{\text {sub }}(z)$ ] be free from unit-circle zeros. If we want the reconstruction itself to be FIR then $\mathbf{R}_{\text {sub }}^{-1}(z)$ has to be FIR which is possible if [det $\mathbf{R}_{\mathrm{sub}}(z)$ ] is a pure delay, i.e.,

$$
\operatorname{det} \mathbf{R}_{\text {sub }}(z)=c z^{-N} \quad \text { (FIR reconstructibility condition) }
$$

for some $c \neq 0$ and some integer $N$.

Example 3: dIn Example 1 we had $F(z)=1+z-z^{2}+z^{3}$ and $M=2$ so that $R_{0}(z)=1-z$ and $R_{1}(z)=1+z$. These polyphase components have unit circle zeros, so we could not reconstruct $x(n)$ from either $x(2 n)$ or $x(2 n-1)$ in a stable manner. Now consider the scheme of Fig. 26 with $L=2$. First we have to find the 2 -fold blocked versions of $R_{0}(z)$ and $R_{1}(z)$. Since

$$
R_{0}(z)=1-z=1+z^{-1} \times\left(-z^{2}\right)
$$

we see that its polyphase components are 1 and $-z$. Similarly those of $R_{1}(z)$ are 1 and $z$. Thus, the $L=2$ blocked versions are

$$
\mathbf{R}_{0}(z)=\left[\begin{array}{cc}
1 & -z \\
-1 & 1
\end{array}\right], \quad \mathbf{R}_{1}(z)=\left[\begin{array}{ll}
1 & z \\
1 & 1
\end{array}\right]
$$




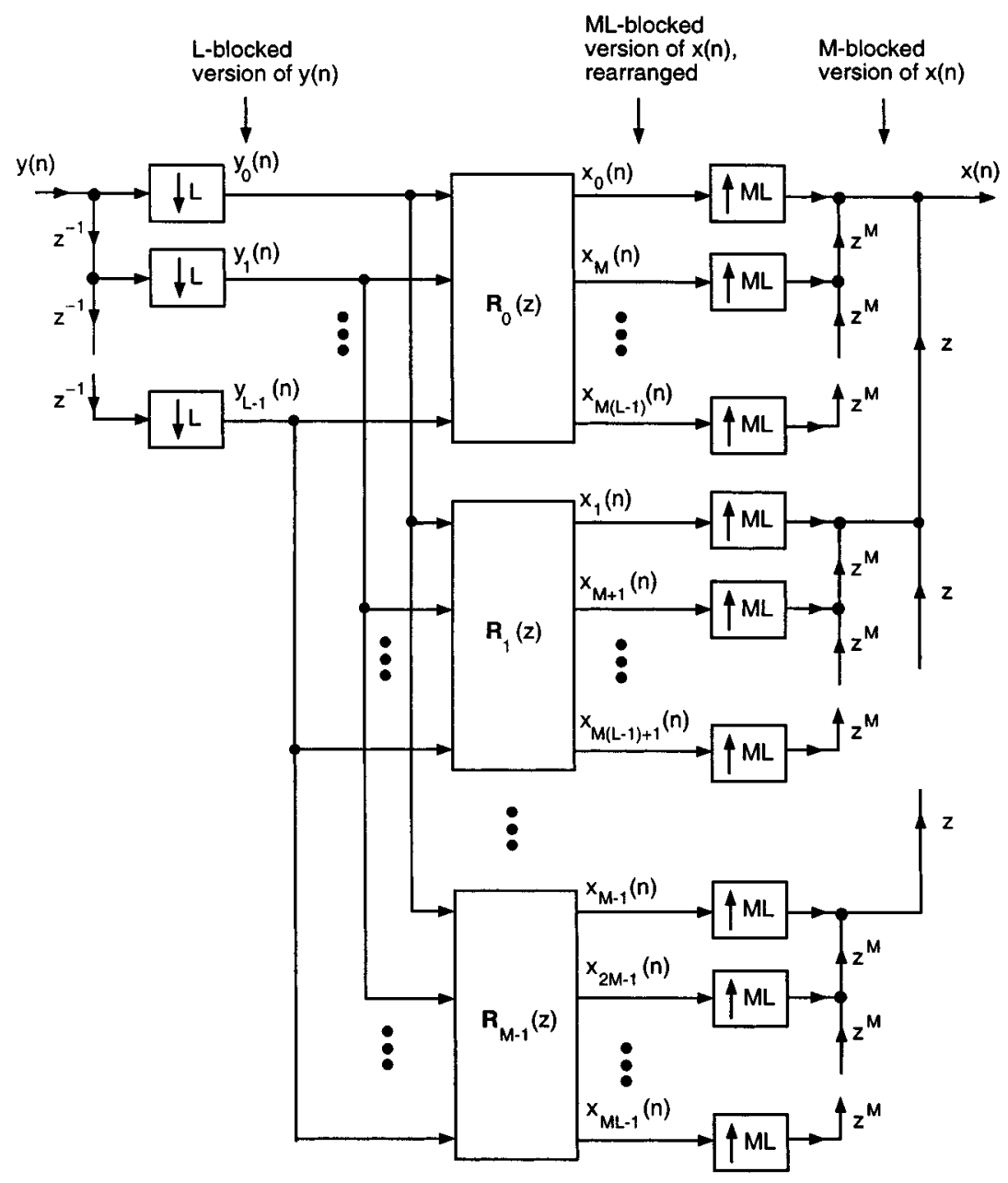

Fig. 26. The $L$-blocked version of the polyphase form shown in Fig. 25.

In the notation of Fig. 26 we have

$$
\left[\begin{array}{l}
X_{0}(z) \\
X_{2}(z) \\
X_{1}(z) \\
X_{3}(z)
\end{array}\right]=\underbrace{\left[\begin{array}{cc}
1 & -z \\
-1 & 1 \\
1 & z \\
1 & 1
\end{array}\right]}_{\mathbf{R}_{\mathrm{big}}(z)}\left[\begin{array}{l}
Y_{0}(z) \\
Y_{1}(z)
\end{array}\right]
$$

Thus $\mathbf{R}_{\text {big }}(z)$ is a $4 \times 2$ matrix. Consider the submatrix

$$
\mathbf{R}_{\text {sub }}=\left[\begin{array}{cc}
-1 & 1 \\
1 & 1
\end{array}\right]
$$

This is a constant nonsingular matrix. This shows that we can write

$$
\left[\begin{array}{l}
Y_{0}(z) \\
Y_{1}(z)
\end{array}\right]=\mathrm{R}_{\mathrm{sub}}^{-1}(z)\left[\begin{array}{l}
X_{2}(z) \\
X_{3}(z)
\end{array}\right]
$$

so that

$$
\begin{aligned}
{\left[\begin{array}{l}
X_{0}(z) \\
X_{2}(z) \\
X_{1}(z) \\
X_{3}(z)
\end{array}\right] } & =\left[\begin{array}{cc}
1 & -z \\
-1 & 1 \\
1 & z \\
1 & 1
\end{array}\right] \mathrm{R}_{\mathrm{sub}}^{-1}(z)\left[\begin{array}{l}
X_{2}(z) \\
X_{3}(z)
\end{array}\right] \\
& =0.5\left[\begin{array}{cc}
1 & -z \\
-1 & 1 \\
1 & z \\
1 & 1
\end{array}\right]\left[\begin{array}{cc}
-1 & 1 \\
1 & 1
\end{array}\right]\left[\begin{array}{l}
X_{2}(z) \\
X_{3}(z)
\end{array}\right]
\end{aligned}
$$

Since $X(z)=X_{0}\left(z^{4}\right)+z X_{1}\left(z^{4}\right)+z^{2} X_{2}\left(z^{4}\right)+z^{3} X_{3}\left(z^{4}\right)$, we finally have

$$
\begin{aligned}
X(z)= & {\left[\begin{array}{llll}
1 & z & z^{2} & z^{3}
\end{array}\right]\left[\begin{array}{l}
X_{0}\left(z^{4}\right) \\
X_{1}\left(z^{4}\right) \\
X_{2}\left(z^{4}\right) \\
X_{3}\left(z^{4}\right)
\end{array}\right] } \\
= & 0.5\left[\begin{array}{llll}
1 & z & z^{2} & z^{3}
\end{array}\right]\left[\begin{array}{cc}
1 & -z^{4} \\
1 & z^{4} \\
-1 & 1 \\
1 & 1
\end{array}\right] \\
& \times\left[\begin{array}{cc}
-1 & 1 \\
1 & 1
\end{array}\right]\left[\begin{array}{l}
X_{2}\left(z^{4}\right) \\
X_{3}\left(z^{4}\right)
\end{array}\right]
\end{aligned}
$$

which simplifies to $X(z)=S_{2}(z) X_{2}\left(z^{4}\right)+S_{3}(z) X_{3}\left(z^{4}\right)$ where

$$
\begin{aligned}
& S_{2}(z)=-0.5\left(1+z-2 z^{2}+z^{4}-z^{5}\right) \\
& S_{3}(z)=0.5\left(1+z+2 z^{3}-z^{4}+z^{5}\right) .
\end{aligned}
$$

Note that $X_{2}(z)$ and $X_{3}(z)$ are the $z$-transforms of $x(4 n-2)$ and $x(4 n-3)$ respectively. So we have shown that $x(n)$ can be reconstructed from $x(4 n-2)$ and $x(4 n-3)$ using the FIR filters $S_{2}(z)$ and $S_{3}(z)$ as shown in Fig. 27(a). That is, we can recover all the samples of $x(n)$ from the nonuniformly decimated version shown by bold lines in Fig. 27(b). 


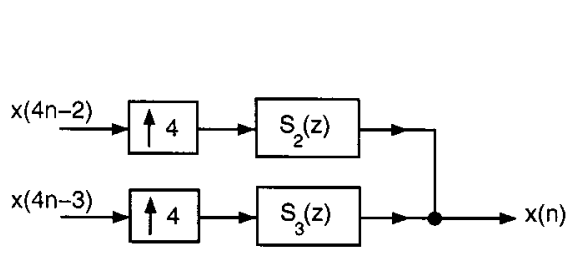

(a)

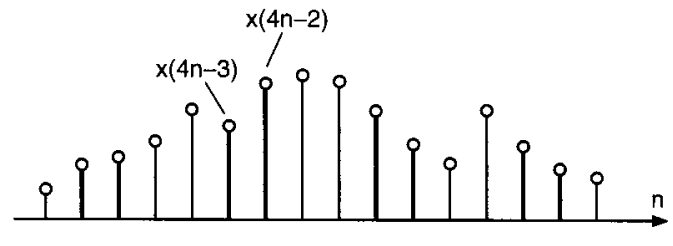

(b)

Fig. 27. Pertaining to Example 3. (a) FIR reconstruction of $x(n)$ from two of its four polyphase components. (b) Interpretation as reconstruction from nonuniformly decimated version.

\section{Conditions for Reconstruction from Nonuniform Decimation}

Note that $\mathbf{R}_{\text {big }}(z)$ depends entirely on the filter $F(z)$ and integers $M$ and $L$ in Fig. 26. With $F(z)$ restricted to be FIR, what is a set of necessary and sufficient conditions for FIR reconstructibility? That is, what are the conditions on $F(z)$ and $M$ so that there will exist an $L$ such that a submatrix $\mathbf{R}_{\text {sub }}(z)$ of $\mathbf{R}_{\text {big }}(z)$ satisfies [det $\mathbf{R}_{\text {sub }}(z)$ ] $=c z^{-N}$ ? This is an open problem. However a set of sufficient conditions has been found [26]:

Theorem 4: Let $x(n)$ be modeled as in Fig. 18. Let $F(z)=\sum_{k=0}^{M-1} z^{k} R_{k}\left(z^{M}\right)$ be FIR and assume there is a pair of polyphase components $R_{i}(z)$ and $R_{j}(z)$ with (a) no multiple zeros and (b) no common zeros. Then, we can recover $x(n)$ from a nonuniformly decimated version with average decimation ratio equal to $M$. Moreover the reconstruction involves only FIR filtering.

We explain the idea of the proof by an example. ${ }^{3}$ Let the two FIR polyphase components $R_{i}(z)$ and $R_{j}(z)$ in Theorem 4 be given by

$$
\begin{aligned}
& R_{i}(z)=1+a_{1} z^{-1}+a_{2} z^{-2} \\
& R_{j}(z)=1+b_{1} z^{-1}+b_{2} z^{-2}+b_{3} z^{-3} .
\end{aligned}
$$

Choose the block length $L$ in Fig. 26 to be the sum of orders of $R_{i}(z)$ and $R_{j}(z)$, i.e., $L=2+3=5$. Then the blocked versions $\mathbf{R}_{i}(z)$ and $\mathbf{R}_{j}(z)$ are

$$
\begin{aligned}
\mathbf{R}_{i}(z) & =\left[\begin{array}{ccccc}
1 & a_{1} & a_{2} & 0 & 0 \\
0 & 1 & a_{1} & a_{2} & 0 \\
0 & 0 & 1 & a_{1} & a_{2} \\
z^{-1} a_{2} & 0 & 0 & 1 & a_{1} \\
z^{-1} a_{1} & z^{-1} a_{2} & 0 & 0 & 1
\end{array}\right] \\
\mathbf{R}_{j}(z) & =\left[\begin{array}{ccccc}
1 & b_{1} & b_{2} & b_{3} & 0 \\
0 & 1 & b_{1} & b_{2} & b_{3} \\
z^{-1} b_{3} & 0 & 1 & b_{1} & b_{2} \\
z^{-1} b_{2} & z^{-1} b_{3} & 0 & 1 & b_{1} \\
z^{-1} b_{1} & z^{-1} b_{2} & z^{-1} b_{3} & 0 & 1
\end{array}\right]
\end{aligned}
$$

As mentioned in Section VI-B these are pseudocirculants. The martrix $\mathbf{R}_{\text {big }}(z)$ is a $10 \times 5$ matrix. Consider the $5 \times 5$ subma-

\footnotetext{
${ }^{3} \mathrm{~A}$ formal proof is mostly a matter of supplying more general notations at the expense of clarity.
}

trix obtained by selecting the first three rows of $\mathbf{R}_{i}(z)$ and first two rows of $\mathbf{R}_{j}(z)$

$$
\mathbf{R}_{\text {sub }}(z)=\left[\begin{array}{ccccc}
1 & a_{1} & a_{2} & 0 & 0 \\
0 & 1 & a_{1} & a_{2} & 0 \\
0 & 0 & 1 & a_{1} & a_{2} \\
1 & b_{1} & b_{2} & b_{3} & 0 \\
0 & 1 & b_{1} & b_{2} & b_{3}
\end{array}\right] .
$$

By choice this matrix is constant (i.e., no $z$ in it). We now claim that this is a nonsingular matrix. The proof depends crucially on the assumptions of the theorem, namely that $R_{i}(z), R_{j}(z)$ have (a) no multiple zeros and (b) no common zeros. Thus, let $1 / a$ and $1 / b$ be the zeros of $R_{i}(z)$ and $1 / d, 1 / e$ and $1 / f$ the zeros of $R_{j}(z)$ (the choice of notations simplify some of the following expressions). Based on these definitions we see that

$$
\begin{aligned}
\mathbf{R}_{\text {sub }}(z)\left[\begin{array}{c}
1 \\
a \\
a^{2} \\
a^{3} \\
a^{4}
\end{array}\right] & =\left[\begin{array}{ccccc}
1 & a_{1} & a_{2} & 0 & 0 \\
0 & 1 & a_{1} & a_{2} & 0 \\
0 & 0 & 1 & a_{1} & a_{2} \\
1 & b_{1} & b_{2} & b_{3} & 0 \\
0 & 1 & b_{1} & b_{2} & b_{3}
\end{array}\right]\left[\begin{array}{c}
1 \\
a \\
a^{2} \\
a^{3} \\
a^{4}
\end{array}\right] \\
& =\left[\begin{array}{c}
R_{i}\left(\frac{1}{a}\right) \\
a R_{i}\left(\frac{1}{a}\right) \\
a^{2} R_{i}\left(\frac{1}{a}\right) \\
R_{j}\left(\frac{1}{a}\right) \\
a R_{j}\left(\frac{1}{a}\right)
\end{array}\right]=\left[\begin{array}{l}
0 \\
0 \\
0 \\
1 \\
a
\end{array}\right] R_{j}\left(\frac{1}{a}\right) .
\end{aligned}
$$

Defining the Vandermonde matrix

$$
\mathbf{V}=\left[\begin{array}{ccccc}
1 & 1 & 1 & 1 & 1 \\
a & b & d & e & f \\
a^{2} & b^{2} & d^{2} & e^{2} & f^{2} \\
a^{3} & b^{3} & d^{3} & e^{3} & f^{3} \\
a^{4} & b^{4} & d^{4} & e^{4} & f^{4}
\end{array}\right]
$$

we therefore see that the product $\mathbf{R}_{\text {sub }}(z) \mathbf{V}$ has the form

$$
\mathbf{R}_{\text {sub }}(z) \mathbf{V}=\left[\begin{array}{ll}
\mathbf{0} & \mathbf{P} \\
\mathbf{Q} & \mathbf{0}
\end{array}\right]
$$

where

$$
\begin{aligned}
& \mathbf{Q}=\left[\begin{array}{ll}
1 & 1 \\
a & b
\end{array}\right]\left[\begin{array}{cc}
R_{j}\left(\frac{1}{a}\right) & 0 \\
0 & R_{j}\left(\frac{1}{b}\right)
\end{array}\right] \text { and } \\
& \mathbf{P}=\left[\begin{array}{lll}
1 & 1 & 1 \\
d & e & f \\
d^{2} & e^{2} & f^{2}
\end{array}\right]\left[\begin{array}{ccc}
R_{i}\left(\frac{1}{d}\right) & 0 & 0 \\
0 & R_{i}\left(\frac{1}{e}\right) & 0 \\
0 & 0 & R_{i}\left(\frac{1}{f}\right)
\end{array}\right] .
\end{aligned}
$$

Since $R_{i}(z)$ and $R_{j}(z)$ do not have multiple zeros or common zeros, the five numbers $a, b, d, e, f$ are distinct. Thus, the Van- 

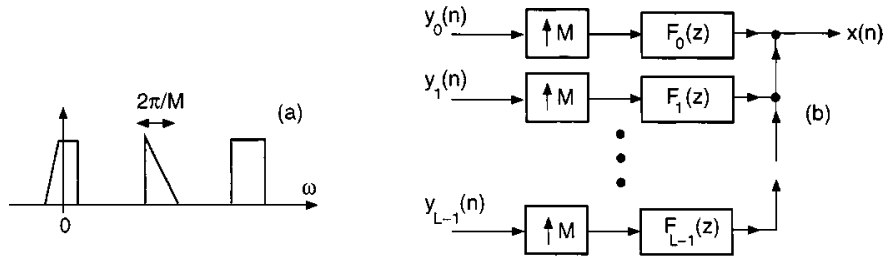

Fig. 28. (a) A signal with several bands of individual width $\leq 2 \pi / M$, and (b) model for such a signal.

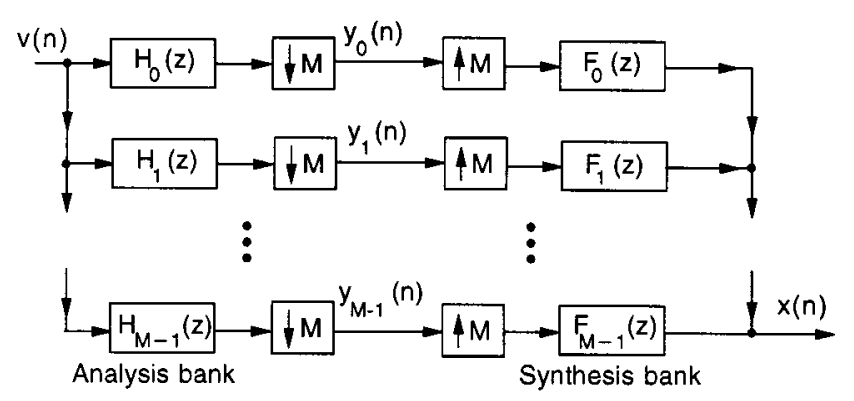

Fig. 29. The maximally decimated filter bank.

dermonde matrix $\mathbf{V}$ is nonsingular. Moreover $\mathbf{P}$ and $\mathbf{Q}$ are nonsingular for the same reason (e.g., $R_{j}(1 / a)$ and $R_{j}(1 / b)$ are nonzero by coprimeness). The matrix $\mathbf{R}_{\text {sub }}(z)$ is therefore nonsingular. Moreover since it is a constant, $\mathbf{R}_{\text {sub }}(z)$ is trivially FIR. This shows that we can reconstruct the components $y_{k}(n)$ in Fig. 26 from first three outputs of $R_{i}(z)$ and first two outputs of $R_{j}(z)$. Thus $y(n)$ (hence $x(n)$ ) can be reconstructed from an $M$-fold nonuniformly decimated version of $x(n)$, using FIR filters.

$\nabla \nabla \nabla$

\section{E. Multiband Models}

Signals which can be approximated by the model of Fig. 18 are typically those which have most energy concentrated in a frequency band of width $2 \pi / M$. For multiband signals with energy concentrated in $L<M$ subbands [Fig. 28(a)] the model of Fig. 28(b) can be used. Subject to minor restrictions (similar to the one-band case) we can reconstruct the signal $x(n)$ in Fig. 28(b) from a nonuniformly decimated version, the average decimation ratio being $M / L>1$ (see [27]). The signal $x(n)$ can be viewed as a partial reconstructions from perfect-reconstruction filter banks [23] of the form shown in Fig. 29. If all the $M$ subbands are retained then $x(n)=v(n)$. If we drop some subbands, then $x(n)$ is a partial reconstruction of $v(n)$. If the filter bank is appropriately designed, then $x(n)$ is the orthogonal projection of $v(n)$ onto the subspace spanned by $L$ of the filters $F_{k}(z)$. Thus orthogonal projections admit reconstruction from subsampled versions, just as bandlimited signals do.

\section{CONCLUDING REMARKS}

The topic of sampling continues to fascinate many researchers in science and engineering. In this paper we reviewed several less known aspects of sampling, with emphasis on non bandlimited signals and stability of reconstruction. For further reading along these lines the reader should study [2], [4], [18] and [19]. Multidimensional versions of some of these results can be interesting especially in the case of nonseparable lattice sampling.

\section{APPENDIX I}

BANDLIMITED Signals: EVALUATING INTEGRALS FROM SUMS

For a $\sigma$-BL signal $x(t)$ it turns out that certain integrals can be calculated exactly by using sums of samples. According to Shannon's sampling theorem

$$
x(t)=\sum_{n=-\infty}^{\infty} x(n T) h(t-n T)
$$

where $T=\pi / \sigma$ and $h(t)=\sin \sigma t / \sigma t$. This $h(t)$ is a lowpass filter with frequency response

$$
H(j \omega)= \begin{cases}T & \text { for }|\omega|<\sigma \\ 0 & \text { elsewhere }\end{cases}
$$

Since $\int h(t) d t=H(j 0)=T$ we see from (29) that $\int x(t) d t=$ $T \sum_{n} x(n T)$. That is

$$
\sum_{n=-\infty}^{\infty} x(n T)=\frac{1}{T} \int_{-\infty}^{\infty} x(t) d t
$$

This shows that the integral of a bandlimited function can be evaluated from the sum of samples taken at any rate above the Nyquist rate. Now the shifted sine functions $h(t-n T)$ constitute an orthogonal basis for $\sigma$-BL functions. That is

$$
\int_{-\infty}^{\infty} h(t-n T) h^{*}(t-m T) d t=T \delta(m-n) .
$$

This can be shown by using the fact that the preceding integral is equal to $T^{2} \int_{-\sigma}^{\sigma} e^{j \omega T(m-n)} d \omega / 2 \pi$ (from Parseval's relation) and simplifying the result. Using this orthogonality it follows from (29) that

$$
\sum_{n=-\infty}^{\infty}|x(n T)|^{2}=\frac{1}{T} \int_{-\infty}^{\infty}|x(t)|^{2} d t
$$

In short, the energy is preserved, that is, energy in the samples is proportional to the energy in $x(t)$. Equations (30) and (32) should in particular be true for the sine function $h(t)=$ $\sin \sigma t / \sigma t$ with sample spacing $T=\pi / \sigma$. In this case these equations yield

$$
\sum_{n} h(n T)=1, \quad \sum_{n} h^{2}(n T)=1
$$

which also follows trivially from the Nyquist $(T)$ property of the sine, namely $h(n T)=\delta(n)$. More generally if a signal has the form (29) for some $h(t)$ (not necessarily bandlimited, e.g., signals in Section II) we see that the integral can be found from the sum of samples as long as $\int_{-\infty}^{\infty} h(t-n T) d t$ is nonzero. Similarly, (32) can be generalized if the functions $\{h(t-n T)\}$ satisfy orthogonality. For an entirely arbitrary integrable signal 


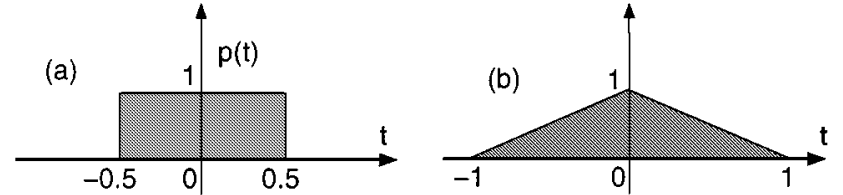

Fig. 30. The pulse and triangle waveforms.

$x(t)(30)$ and (32) can only be approximatey true, the approximation typically improving with the sampling rate.

\section{APPENDIX II}

\section{MATHEMATICAL IDENTITIES FROM SAMPLING THEORY}

It turns out that several standard math identities can be derived from a basic knowledge of the simplest form of sampling theory. Consider the pulse function $p(t)$ shown in Fig. 30(a) whose Fourier transform is

$$
P(j \omega)=\frac{\sin \left(\frac{\omega}{2}\right)}{\frac{\omega}{2}} .
$$

If this is sampled with spacing $T=1$ the result is $x[n]=\delta[n]$, so $X\left(e^{j \omega}\right)=1$. But sampling theory also says that $X\left(e^{j \omega}\right)=$ $\sum_{k} P(j(\omega+2 \pi k))$, which proves

$$
\sum_{k=-\infty}^{\infty} \frac{\sin \left(\frac{\omega+2 \pi k}{2}\right)}{\frac{\omega+2 \pi k}{2}}=1
$$

for all $\omega$. For example, suppose $\omega=\pi$, then this yields

$$
\sum_{k=-\infty}^{\infty} \frac{\sin \frac{(2 k+1) \pi}{2}}{2 k+1}=\frac{\pi}{2}
$$

That is, $\sum_{\text {odd } m} \sin (\pi m / 2) / m=\pi / 2$ from which ${ }^{4}$ we get the well-known identity

$$
1-\frac{1}{3}+\frac{1}{5}-\frac{1}{7}+\frac{1}{9}+\cdots=\frac{\pi}{4}
$$

More generally, since $\sin ((\omega+2 \pi k) / 2)=\sin (\omega / 2+k \pi)=$ $(-1)^{k} \sin (\omega / 2)$ we obtain from (35) the identity

$$
\sum_{k=-\infty}^{\infty} \frac{(-1)^{k}}{\omega+2 \pi k}=\frac{1}{2 \sin \left(\frac{\omega}{2}\right)}, \quad 0<\omega<2 \pi .
$$

Next, consider the triangle signal in Fig. 30(b), obtained by convolving the pulse with itself. This has the Fourier transform $P^{2}(j \omega)$. If this is sampled we again get $x[n]=\delta[n]$, so $X\left(e^{j \omega}\right)=1$ again. But according to sampling theory $X\left(e^{j \omega}\right)=$ $\sum_{k} P^{2}(j(\omega+2 \pi k))$, so

$$
\sum_{k=-\infty}^{\infty} \frac{\sin ^{2}\left(\frac{\omega+2 \pi k}{2}\right)}{\frac{(\omega+2 \pi k)^{2}}{4}}=1 .
$$

By setting $\omega=\pi$ we get the identity

$$
1+\frac{1}{3^{2}}+\frac{1}{5^{2}}+\frac{1}{7^{2}}+\frac{1}{9^{2}}+\cdots=\frac{\pi^{2}}{8}
$$

and more generally, the identity $\sum_{k=-\infty}^{\infty} 1 /(\omega+2 \pi k)^{2}=$ $1 / 4 \sin ^{2}(\omega / 2)$, for $0<\omega<2 \pi$.

\footnotetext{
${ }^{4}$ Another way to arrive at this identity by starting from the FT of the bandlimited sine function was indicated in a Caltech lecture several years ago by the late Prof. E. C. Posner. In fact, the material in Appendix II is inspired by that lecture.
}

\section{REFERENCES}

[1] A. N. Akansu and R. A. Haddad, Multiresolution Signal Decomposition. New York: Academic, 1992.

[2] A. Aldroubi and M. Unser, "Sampling procedures in function spaces and asymptotic equivalence with Shannon's sampling theory," Numer. Funct. Anal. Optim., vol. 15, pp. 1-21, 1994.

[3] I. Daubechies, Ten Lectures on Wavelets, ser. CBMS. Philadelphia, PA: SIAM, 1992.

[4] I. Djokovic and P. P. Vaidyanathan, "Generalized sampling theorems in multiresolution subspaces," IEEE Trans. Signal Processing, vol. 45, pp. 583-599, Mar. 1997.

[5] Y. C. Eldar and A. V. Oppenheim, "Filter bank reconstruction of bandlimited signals from nonuniform and generalized samples," IEEE Trans. Signal Processing, vol. 48, pp. 2864-2875, Oct. 2000.

[6] A. J. Jerri, "The Shannon sampling theorem-its various extensions and applications: a tutorial review," Proc. IEEE, pp. 1565-1596, Nov. 1977.

[7] D. G. Luenberger, Optimization by Vector Space Methods. New York: Wiley, 1969.

[8] S. Mallat, "A theory for multiresolution signal decomposition: the wavelet representation," IEEE Trans. Pattern Anal. Machine Intell., pp. 674-693, July 1989.

[9] H. Nyquist, "Certain topics in telegraph transmission theory," IEE Trans., vol. 47, pp. 617-644, Jan. 1928.

[10] A. V. Oppenheim, A. S. Willsky, and I. T. Young, Signals and Systems. Englewood Cliffs, NJ: Prentice-Hall, 1983.

[11] W. Rudin, Real and Complex Analysis. New York: McGraw-Hill, 1966.

[12] I. J. Schoenberg, Cardinal Spline Interpolation . Philadelphia, PA: SIAM, 1973

[13] C. E. Shannon, "Communications in the presence of noise," Proc. IREE, vol. 37, pp. 10-21, Jan. 1949.

[14] M. Unser, A. Aldroubi, and M. Eden, "Fast $B$-spline transforms for continuous image representation and interpolation," IEEE Trans. Pattern Anal. Machine Intell., pp. 277-285, Mar. 1991.

[15] M. Unser and A. Aldroubi, "Polynomial splines and wavelets," in Wavelets, A Tutorial in Theory and Applications, C. K. Chui, Ed. New York: Academic, 1992

[16] M. Unser, A. Aldroubi, and M. Eden, " $B$-spline signal processing: Part I-theory,” IEEE Trans. Signal Processing, vol. 41, pp. 821-833, Feb. 1993.

[17] — , " $B$-spline signal processing: Part II—efficient design and applications," IEEE Trans. Signal Processing, vol. 41, pp. 834-848, Feb. 1993.

[18] M. Unser and J. Zerubia, "Generalized sampling without bandlimiting constraints," in Proc. IEEE Int. Conf. Acoustics, Speech, and Signal Processing, Munich, Germany, Apr. 1997, pp. 2113-2116.

[19] _ _ "Generalized sampling: stability and performance analysis," IEEE Trans. Signal Processing, vol. 45, pp. 2941-2950, Dec. 1997.

[20] M. Unser, "Sampling-50 years after Shannon," Proc. IEEE, vol. 88, pp. 569-587, Apr. 2000.

[21] P. P. Vaidyanathan and S. K. Mitra, "Polyphase networks, block digital filtering, LPTV systems, and alias-free QMF banks: a unified approach based on pseudocirculants," IEEE Trans. Acoust., Speech, Signal Processing, vol. 36, pp. 381-391, Mar. 1988.

[22] P. P. Vaidyanathan and V. C. Liu, "Classical sampling theorems in the context of multirate and polyphase digital filter bank structures," IEEE Trans. Acoust., Speech, Signal Processing, vol. 36, pp. 1480-1495, Sept. 1988

[23] P. P. Vaidyanathan, Multirate Systems and Filter Banks. Englewood Cliffs, NJ: Prentice-Hall., 1993.

[24] _ - "Sampling theorems from wavelet and filter bank theory," in Mircosystems Technology for Multimedia Applications: an Introduction, B. Sheu, B. Ismail, E. Sanchez-Sinencio, and T. H. Wu, Eds. Piscataway, NJ: IEEE Press, 1995.

[25] P. P. Vaidyanathan and I. Djokovic, "Wavelet transforms," in The Circuits and Filters Handbook, W. K. Chen, Ed. Boca Raton, FL: CRC , 1995, pp. 134-219.

[26] P. P. Vaidyanathan and S.-M. Phoong, "Reconstruction of sequences from nonuniform samples," in Proc. IEEE Int. Symp. Circuits and Systems, Seattle, Washington, Apr.-May 1995, pp. 601-604.

[27] — - "Discrete time signals which can be recovered from samples," in Proc. IEEE Int. Conf. Acoustics, Speech, and Signal Processing, Detroit, MI, May 1995, pp. 1448-1451.

[28] P. P. Vaidyanathan and B. Vrcelj, "Biorthogonal Partners and Applications," IEEE Trans. Signal Process., vol. 49, pp. 1013-1027, May 2001.

[29] G. G. Walter, "A sampling theorem for wavelet subspaces," IEEE Trans. Inform. Theory, pp. 881-884, Mar. 1992. 


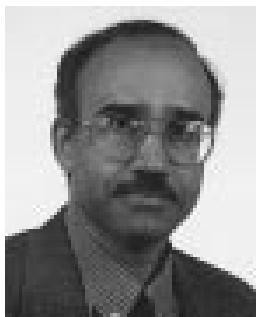

P. P. Vaidyanathan (S'80-M'83-SM'88-F'91) was born in Calcutta, India in 1954. He received the B.Sc. (Hons.) degree in physics and the B.Tech. and M.Tech. degrees in radiophysics and electronics, all from the University of Calcutta, India, in 1974, 1977, and 1979, respectively, and the Ph.D. degree in electrical and computer engineering, from the University of California at Santa Barbara, in 1982.

He was a postdoctoral fellow at the University of California, Santa Barbara from September 1982 to March 1983. In March 1983, he joined the electrical engineering department of the California Institute of Technology, Pasadena, as an Assistant Professor, and since 1993, has been Professor of electrical engineering there. His main research interests are in digital signal processing, multirate systems, wavelet transforms and signal processing for digital communications.

Dr. Vaidyanathan served as Vice-Chairman of the Technical Program Committee for the 1983 IEEE International Symposium on Circuits and Systems, and as the Technical Program Chairman for the 1992 IEEE International Symposium on Circuits and Systems. He was an Associate Eeditor for the IEEE Transactions on Circuits and Systems for the period 1985-1987, and is currently an Associate Editor for the IEEE SigNAL PROCESSING LETTERS, and a consulting editor for the Applied and Computational Harmonic Analysis. He has been a Guest Editor in 1998, for special issues of the IEEE TRANSACTIONS ON SignAL PROCESSING and the IEEE TRANSACTIONS ON CIRCUITS AND SYSTEMS II, on the topics of filter banks, wavelets, and subband coders. He has authored a number on papers in IEEE journals, and is the author of the book Multirate Systems and Filter Banks (Prentice Hall: Englewood Cliffs, NJ). He has written several chapters for various signal processing handbooks. He was a recepient of the Award for excellence in teaching at the California Institute of Technology for the years 1983-1984, 1992-1993, and 1993-1994; the NSF's Presidential Young Investigator award in 1986; the IEEE ASSP Senior Award for his paper on multirate perfect-reconstruction filter banks, in 1989; the S. K. Mitra Memorial Award from the Institute of Electronics and Telecommunications Engineers, India, for his joint paper in the IETE journal, in 1990; and the 1995 F. E. Terman Award of the American Society for Engineering Education, sponsored by Hewlett Packard, for his contributions to engineering education; He was chosen a distinguished lecturer for the IEEE Signal Processing Society for the year 1996-1997; was chosen to receive the IEEE CAS Society's Golden Jubilee Medal in 1999; and was the coauthor of a paper on linear-phase perfect reconstruction filter banks in the IEEE TRANSACTIONS ON SIGNAL PROCESSING, for which the first author (Truong Nguyen) received the Young Outstanding Author award in 1993. Dr. Vaidyanathan has given several plenary talks including those at the Eusipco'98, and SPCOM'95 conferences on signal processing. 